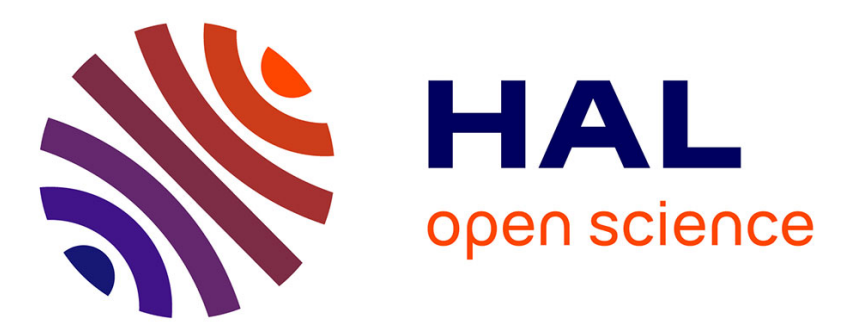

\title{
Prediction of limit cycle amplitudes in thermoacoustic engines by means of impedance measurements
}

Valentin Zorgnotti, Guillaume Penelet, Gaëlle Poignand, Steven Garrett

\section{To cite this version:}

Valentin Zorgnotti, Guillaume Penelet, Gaëlle Poignand, Steven Garrett. Prediction of limit cycle amplitudes in thermoacoustic engines by means of impedance measurements. Journal of Applied Physics, 2018, 124 (15), pp.154901. 10.1063/1.5040906 . hal-02057067

\section{HAL Id: hal-02057067 \\ https://hal-univ-lemans.archives-ouvertes.fr/hal-02057067}

Submitted on 5 Mar 2019

HAL is a multi-disciplinary open access archive for the deposit and dissemination of scientific research documents, whether they are published or not. The documents may come from teaching and research institutions in France or abroad, or from public or private research centers.
L'archive ouverte pluridisciplinaire HAL, est destinée au dépôt et à la diffusion de documents scientifiques de niveau recherche, publiés ou non, émanant des établissements d'enseignement et de recherche français ou étrangers, des laboratoires publics ou privés. 
This manuscript was accepted by J. Appl. Phys. Click here to see the version of record.

V. Zorgnotti, ${ }^{1, \text { a) }}$ G.Penelet, ${ }^{1}$ G. Poignand ${ }^{1}$ and S.L. Garrett ${ }^{1}$

Laboratoire d'Acoustique de l'Université du Mans (UMR 6613 CNRS), avenue Olivier Messiaen, 72085 Le Mans Cedex 9, FRANCE

(Dated: 28 July 2018)

This paper deals with the prediction of the frequency and the amplitude of selfsustained oscillations generated in thermoacoustic prime movers, which are compared to measurements. A specially designed, high amplitude, acoustic impedance sensor was developed to perform measurements of the input impedance of a thermoacoustic core, as a function of the heating power supplied to the device, of the frequency, and of the amplitude of acoustic forcing. Those measurements can then be used to predict the spontaneous generation of acoustic oscillations and their saturation up to a steady-state. Those predictions were successful for various acoustic loads connected to the thermoacoustic core. Moreover, the measurements of acoustic impedance as a function of the amplitude of acoustic oscillations are compared to a model based on the linear thermoacoustic theory, and this comparison provides insights into the processes controlling the saturation of acoustic oscillations. The experimental procedure described in this paper can also have practical value, since it provides an empirical way, in principle, to optimize the coupling between the thermoacoustic core and the load, so that the potential efficiency of thermoacoustic energy conversion is maximized.

a) Electronic mail: valentin.zorgnotti@univ-lemans.fr 
This manuscript was accepted by J. Appl. Phys. Click here to see the version of record.

Heat addition, which is one of the elementary sources of sound production ${ }^{1}$, is employed for the purpose of energy conversion in devices called thermoacoustic engines. The basic elements of these devices are an acoustic resonator and a porous medium inside, usually referred to as a stack. Heat addition is employed to maintain a temperature gradient along the stack, and this leads (above a critical value of the temperature gradient) to the spontaneous generation of high amplitude acoustic oscillations at the frequency of the most unstable acoustic mode. This transduction process, which occurs within thermal boundary layers in the stack, can be used for the delivery of mechanical power to a load, giving rise to a thermoacoustic engine ${ }^{2}$.

Thermoacoustic engines have been studied for about three decades ${ }^{3}$, and because of their inherent simplicity and their good performances ${ }^{4-}$, they represent nowadays credible alternatives to other kinds of thermodynamic engines ${ }^{2}$, e.g. for applications devoted to the recovery of waste heat ${ }^{8}$. Although much has been done in terms both of engine developments and basic understanding of their operation, further efforts are still needed to promote their applications. Among the key points which still require further research are the accurate description of sound propagation and heat transport through stack/regenerators having complex geometries (e.g. metallic foams or stacked meshgrids ${ }^{9,10}$ ), the accurate description of both entropic and hydrodynamic entrance effects involved at the ends of the stack and of the heat exchangers ${ }^{11-13}$, or the proper account of nonlinear effects like acoustic streaming ${ }^{14,15}$ or higher harmonics generation ${ }^{16}$. It is indeed worth pointing out that although various design tools $^{17,18}$ based on Rott's linear theory of thermoacoustics ${ }^{19}$ are available, the accurate prediction of a limit cycle amplitude (or even its stability) remains, sometimes ${ }^{20}$, an important issue.

This paper aims at evaluating a new way of characterizing the operation of a thermoacoustic prime mover, which is based on a "black-box" approach, or in other words on the characterization of the thermoacoustic core as a whole, from experiments. Similar approaches have already been used but were actually limited to low amplitudes of acoustic pressure: Guedra et al. ${ }^{10,21}$ as well as Bannwart et al. ${ }^{22}$ have shown that the measurements of the acoustic transfer matrices of a thermoacoustic core (within a given range of heat supply and frequency) can be used to predict the onset of the thermoacoustic instability for various en- 
Publishigiges equipped with the thermoacoustic core characterized beforehand. A similar approach has been used by Hatori et al. ${ }^{23}$ who have shown that acoustic impedance measurements can be used to define the optimum load that should be connected to the thermoacoustic core, so that a minimum temperature gradient is required to trigger self-sustained oscillations. The main advantage of these approaches is that once the thermoacoutic core is characterized in terms of a two-port network ${ }^{21,22}$ or a dipole ${ }^{23}$ for various amounts of heating and various frequencies, there is no need to describe heat transport and wave propagation through this element. It is then quite straightforward to evaluate the optimum acoustic state at its boundaries giving rise to maximum thermoacoustic amplification. This amounts to solving an eigenvalue problem, as shown by Holzinger et al.4. However, all the studies mentioned above were limited to the linear regime where the impact of high amplitude acoustic oscillations on the mean temperature field (e.g. due to acoustic streaming or to thermoacoustic heat transport within the stack) and on power dissipation (e.g. due to the generation of vorticity close to the stack ends) were not considered. Due to these omissions, the use of experimental data is necessarily limited to the prediction of the onset threshold of the thermoacoustic instability, since no saturation mechanism is taken into account.

In this paper, we present an extension of these works to the regime of large amplitude acoustic oscillations. The basic idea consists of measuring the acoustical input impedance of a thermoacoustic core as a function of the supplied heat power, of the frequency, and of the sound pressure level (imposed by the acoustic impedance sensor). We show that the measurements can be used to predict the amplitude and the frequency of steady-state acoustic oscillations resulting from the (resonant) coupling of the thermoacoustic core to an acoustic load. In section II, the experimental test-bench is presented and attention is focused on its calibration procedure. In section III, various results of such impedance measurements are presented and compared to a model based on Rott's theory ${ }^{19}$ combining a description of sound amplification by temperature gradients, and of sound saturation due to thermoacoustic heat transport: this model is described in Appendix A. We also show that the experimental results can be used to predict steady-states, and that the predicted steady-states match with the ones obtained in experiments. Finally, conclusions are drawn in section IV, where possible extensions of these works are also discussed. 
(a)

Coupling Duct

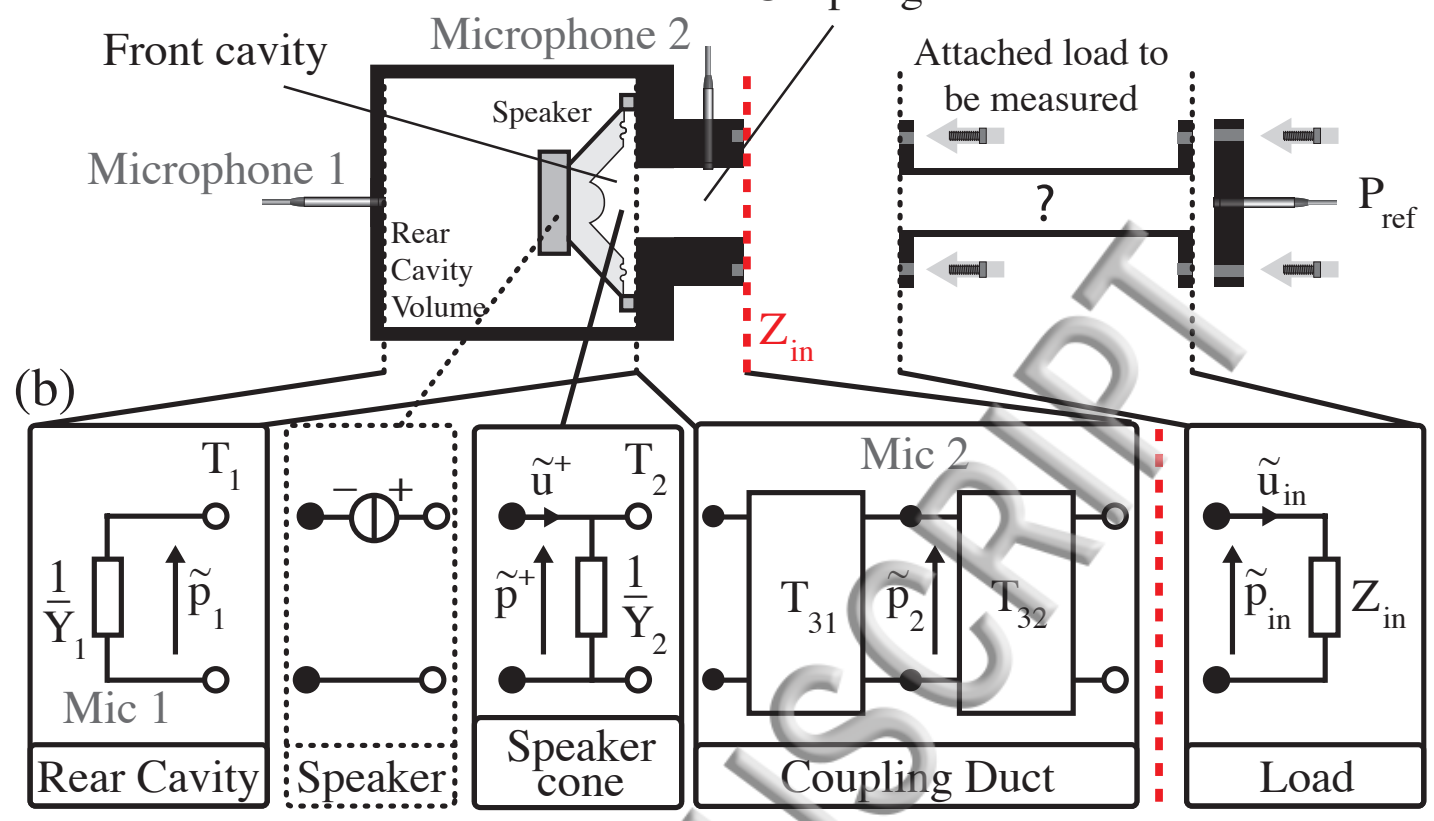

FIG. 1. (a) Sketch of the experimental set-up for acoustic impedance measurements. The impedance sensor consists of a moving coil loudspeaker mounted inside a cavity. The input impedances are computed (from the two microphone signals) at the red dashed line location, i.e. at the sensor output, where any load can be inserted. (b) This set-up is described as an equivalent electric circuit. The rear cavity and the volume of fluid facing the speaker cone are represented as equivalent admittances. The coupling duct, along which Mic. 2 is mounted flush, is represented as two two-port networks with their associated transfer matrices $T_{31}$ and $T_{32}$. The microphone labeled as $P_{\text {ref }}$ is an additionnal microphone used to measure the sound pressure level into the load.

\section{APPARATUS}

\section{A. Experimental set-up}

A sketch of the experimental set-up used for acoustic impedance measurements is presented in Fig. 1(a): it is a high-amplitude acoustic impedance sensor ${ }^{25,26}$ developed especially for this study which allows measurement of the ratio of acoustic pressure to acoustic volume velocity, at the entrance of any acoustic load attached to the sensor. The measured impedance is therefore the input impedance of the load. This device consists of a moving coil loudspeaker (Beyma 6MI100) mounted in a rear cavity, and connected to the load through 
Publishia giront cavity and a coupling duct. A microphone (Mic 1, see Fig. 1) is flush-mounted in the rear cavity to measure the acoustic pressure $p_{1}(t)$, while another microphone (Mic 2, see Fig. 1) measures the acoustic pressure $p_{2}(t)$ in the coupling duct. The front cavity is essentially a gas spring and it corresponds to the volume of fluid facing the loudspeaker's front cone. It is connected to the coupling duct, whose diameter matches the diameter of the acoustic load. The two microphone signals are used to compute the input impedance of the attached load at a location marked with a red dashed line, at the sensor output, in Fig. 1(a), as explained below. An electroacoustic analog of the-experimental test-bench is also presented in Fig. 1(b), which helps understanding how the impedance can be calculated from the measurements of the acoustic pressures $p_{1}(t)$ and $p_{2}(t)$. The computation process is based on the accurate knowledge of the system acoustic behavior and the geometry of the apparatus.

The lumped-element model of Fig. 1(b) represents the rear and front cavities as two complex admittances $Y_{1}$ and $Y_{2}$ which are determined from the calibration procedure described in the next sub-section. The coupling duct used for the connection to the load (and for the measurement of the acoustic pressure $p_{2}$ ) is described by means of two-port networks, whose corresponding transfer matrices, $T_{31}$ and $T_{32}$, can be determined as far as the lossy propagation of plane waves through each piece of duct is assumed. More precisely, each matrix can be derived from the formula

$$
T=\left[\begin{array}{cc}
\cos (k L) & -i Z_{c} \sin (k L) \\
-i Z_{c}^{-1} \sin (k L) & \cos (k L)
\end{array}\right],
$$

where L stands for the length of the duct, and where $k$ and $Z_{c}$ respectively stand for the complex wave number and the characteristic impedance of the duct [e.g. see ref ${ }^{22}$, Eqs. (14) and (15)]. Therefore, if the admittances $Y_{i}$ and the T-matrices $T_{31}$ and $T_{32}$ are known, it is almost straightforward to determine the input impedance $Z_{i n}$ as a function of the angular frequency $\omega$ and of the frequency response function defined as $H_{21}=\tilde{p}_{2} / \tilde{p}_{1}$ where $\tilde{p}_{1}$ and $\tilde{p}_{2}$ stand for the complex amplitudes of acoustic pressure measured by the two microphones [these complex amplitudes are defined from the fluctuating pressures $p_{i}(t)$ as $\left.p_{i}(t)=\Re\left(\tilde{p}_{i} e^{i \omega t}\right)\right]$. When the sensor executes harmonic oscillations at angular frequency $\omega$ and is connected to an arbitrary load, the input acoustic impedance $Z_{i n}=\tilde{p}_{i n} / \tilde{u}_{i n}$ is defined as the ratio between the complex amplitude of acoustic pressure $\left(\tilde{p}_{i n}\right)$ and the complex amplitude of volume velocity $\left(\tilde{u}_{i n}\right)$ entering into the load. The pressure $\tilde{p}_{i n}$ and the volume 
Publishing city $\tilde{u}_{i n}$ can be written as functions of the pressure $\tilde{p}^{+}$and volume velocity $\tilde{u}^{+}$just in front of the loudspeaker membrane:

$$
\left\{\begin{array}{l}
\tilde{p}_{i n} \\
\tilde{u}_{i n}
\end{array}\right\}=\mathbf{T}_{32} \times \mathbf{T}_{31} \times \mathbf{T}_{2} \times\left\{\begin{array}{l}
\tilde{p}^{+} \\
\tilde{u}^{+}
\end{array}\right\},
$$

where $\mathbf{T}_{32}$ and $\mathbf{T}_{31}$ are the $\mathrm{T}$-matrices describing wave propagation through the coupling duct, and where

$$
\mathbf{T}_{2}=\left\{\begin{array}{cc}
1 & 0 \\
-Y_{2} & 1
\end{array}\right\}
$$

corresponds to the transfer matrix of the front cavity (i.e. from the membrane to the entrance of the coupling duct). The volume velocity provided by the loudspeaker $\tilde{u}^{+}$can be expressed as a function of the pressure measured by Mic. 1 and of the admittance $Y_{1}$ of the rear cavity:

$$
\tilde{u}^{+}=-Y_{1} \tilde{p}_{1}
$$

while the acoustic pressure $\tilde{p}^{+}$can be expressed as a function of $\tilde{u}^{+}$(and therefore of $\tilde{p}_{1}$ ) and of the measured pressure $\tilde{p}_{2}$ through the transfer matrix $T_{2}$ of the front cavity and the transfer matrix $T_{31}$. As a result, an explicit relation between the input impedance $Z_{\text {in }}$ and the frequency response function $H_{21}$ is obtained, which involves the transfer matrices $T_{31}$ and $T_{32}$ of the coupling duct, and the admittances $Y_{1,2}$ of the front and the rear cavities. However, an exact estimation of the latter admittances $Y_{1,2}$ can not be obtained analytically, and therefore it has to be estimated experimentally from a preliminary calibration procedure. This procedure is described in the following sub-section.

\section{B. Calibration procedure}

The accuracy of the measurements relies on the quality of the calibration of the system, which involves a good relative calibration of the two microphones and an accurate knowledge of the admittances $Y_{1}$ and $Y_{2}$. Both the rear and the front cavities mainly act as gas compliances, but these compliances are not easy to determine from the dimensions of the system, and there are also some losses in the cavities which must be taken into account. Therefore, the admittances $Y_{1}$ and $Y_{2}$ must be determined from experiments as functions of the frequency, and this is done by means of a two-load calibration procedure ${ }^{25,26}$. 
Publishing Two measurements are made, at low amplitude of forcing, with two different loads connected to the sensor. One load, denoted as $Z_{\text {load }}^{(R)}$, is a rigid plate and a first set of measurements $H_{21}^{(R)}(\omega)$ is obtained. The second load, denoted as $Z_{\text {load }}^{(D)}$ is an empty duct of length $L=20 \mathrm{~cm}$ and inner radius $R=2.7 \mathrm{~cm}$ which is terminated by a rigid plug: this leads to a second set of measurements $H_{21}^{(D)}(\omega)$. Because the corresponding impedances of the two loads are known from theory, they can be used as references so that the two sets of measurements enable determination of the two unknown admittances $Y_{1}$ and $Y_{2}$ as functions of the frequency. After manipulation, this leads to the following relations:

$$
\begin{gathered}
Y_{1}=\frac{1}{Z_{2}^{(R)} H_{21}^{(R)}\left(\beta^{(R)}+\widehat{(R)} Y_{2}\right)} \\
Y_{2}=\frac{Z_{2}^{(R)} \beta^{(D)} H_{21}^{(D)}-Z_{2}^{(D)} \beta^{(R)} H_{21}^{(R)}}{Z_{2}^{(D)} \gamma^{(R)} H_{21}^{(R)}-Z_{2}^{(R)} \gamma^{(D)} H_{21}^{(D)}} .
\end{gathered}
$$

where the frequency dependent parameters $\beta$ and $\gamma$ are defined as

$$
\begin{gathered}
\beta^{(R, D)}=-Z_{2}^{(R, D)} \mathbf{T}_{31}(2,1)+\mathbf{T}_{31}(1,1), \\
\gamma^{(R, D)}=Z_{2}^{(R, D)} \mathbf{T}_{31}(2,2)-\mathbf{T}_{31}(1,2) .
\end{gathered}
$$

$T_{31}(i, j)$ stands for the coefficient of the $i^{\text {th }}$ row and $j^{\text {th }}$ column of the transfer matrix $T_{31}$, and

$$
Z_{2}^{(R, D)}=\frac{\mathbf{T}_{32}(2,2) Z_{\text {load }}^{(R, D)}-\mathbf{T}_{32}(1,2)}{\mathbf{T}_{32}(1,1)-\mathbf{T}_{32}(2,1) Z_{\text {load }}^{(R, D)}}
$$

corresponds to the impedance at the position of Mic. 2, which itself depends on the reference load, $Z_{\text {load }}^{(R)}$ or $Z_{\text {load }}^{(D)}$. Once the two admittances $Y_{1,2}$ are determined, the quality of the calibration is checked by measuring a third known load, e.g. an empty duct with a different length terminated with a rigid plug, and by comparing the measured load with its theoretical value.

Because the objective is also to measure the input impedance as a function of the amplitude of forcing (with acoustic pressure amplitudes ranging from a few tens of Pa up to around $1300 \mathrm{~Pa}$ in the following), the possible dependence of $Y_{1,2}$ with the acoustic pressure amplitude due to nonlinear losses within the impedance sensor must be considered. This is done by fixing the angular frequency and by repeating the two-loads calibration procedure described above to determine the variations of $Y_{1}$ and $Y_{2}$ as functions of the pressure amplitude $\left|\tilde{p}_{1}\right|$ in the rear cavity. Such a nonlinear calibration procedure is however based on 


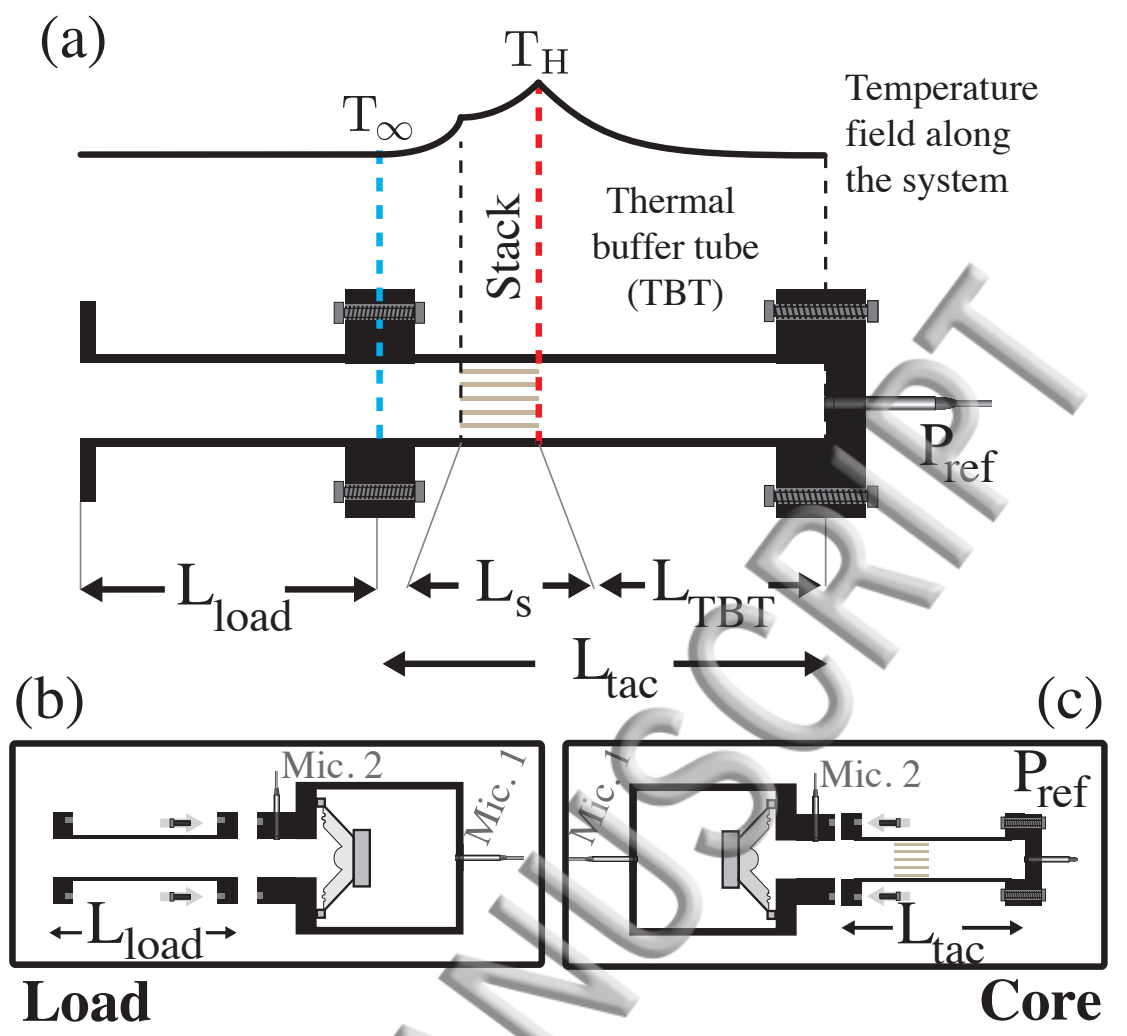

FIG. 2. (a) Schematic representation of the thermoacoustic engine. It is composed of an empty duct (the load) connected to the thermo-acoustic core (TAC) inside which is placed the stack subjected to a temperature gradient. The left-side of the load can either be open or closed by a rigid plug. (b) The input impedance of the load is measured as a function of the frequency. (c) The input impedance of the thermoacoustic core is measured as a function of the heat power supply, of the frequency, and of the amplitude of acoustic forcing.

the assumption that the impedances of the two reference loads do not depend on the sound pressure level, which might not be the case (especially for $Z_{\text {load }}^{(D)}$ ) because of nonlinear propagation effects ${ }^{2}$. As will be shown in the following, the accuracy of the results presented in section III seem to confirm that this approximation remains acceptable as other sources of errors play a more significant role.

\section{Basic principles of the measurement procedure}

The system under consideration in this study is the one presented in Fig. 2(a), which consists of a standing-wave thermoacoustic prime mover. It is basically composed of an acoustic 
Publishiregonator with a porous medium inside, referred to as the stack ${ }^{3}$, and the application of a temperature gradient along the stack is used to trigger self-sustained resonant oscillations. The goal of this study is to make use of the impedance sensor that has been described to predict the onset of acoustic oscillations as well as the amplitude and the frequency of those steady-state oscillations. To that purpose, the basic idea is to make use of impedance matching between two sub-parts of the engine, which are characterized beforehand using the impedance sensor (see Fig. 2(b-c)).

The first sub-part of the engine, denoted as the load, is a duct with a variable length $L_{\text {load }}$ and inner radius $R_{\text {load }}=2.7 \mathrm{~cm}$ filled with air at 1 bar and room temperature $T_{\infty}$. It can be either closed by a rigid plug or open to free space (it could also be connected to a mechanical load like a loudspeaker). The load does not contribute directly to the thermoacoustic amplification of sound waves, but it plays a key role in the potential triggering of self-sustained oscillations since its coupling with the other sub-part determines both the eigen frequencies and the associated spatial distribution of the acoustic field. The second sub-part of the engine, i.e. the thermoacoustic core (TAC), consists of a duct with length $L_{t a c}=34 \mathrm{~cm}$ and inner radius $R_{t a c}=R_{\text {load }}$, terminated with a rigid plug. Inside this duct, the stack of length $L_{s}=4 \mathrm{~cm}$ is inserted. The tube that is formed by the remaining part of the core, between the stack end and the rigid plug is called the thermal buffer tube (TBT) and it is $22 \mathrm{~cm}$ long in this study. It consists of a 400 CPSI (Cells Per Square Inch) Celcor ${ }^{\circledR}$ ceramic material made of many parallel square channels running along the duct's axis. The role of the stack is to promote the thermoacoustic amplification process which occurs within thermal boundary layers in each channel ${ }^{3}$. Heat is supplied on the right side of the stack by means of a nichrome wire $(50 \mathrm{~cm}$ in length, $0.25 \mathrm{~mm}$ in diameter) which is rolled through the stack end. This wire is connected to a DC power supply, so that the temperature gradient is created because of the heat leaks through the lateral walls and the left end of the stack is not equiped with a cold heat exchanger (see Fig. 2). Two thermocouples are placed in the thermoacoustic core: a first one gives the temperature $\mathrm{T}_{\text {hot }}$ along the duct axis close to the Nichrome wire, and a second thermocouple measures the temperature $\mathrm{T}_{\text {in }}$ at the input of the thermoacoustic core. These two measured temperatures are only used as indicators of the temperature field in the system (notably to check that the temperature $\mathrm{T}_{\text {in }}$ remains close to the room temperature $T_{\infty}$ ) which is not uniform through the duct's cross-sectional area. 
PublishingAs depicted schematically in Fig. 2(b-c), the experiments performed in this study consist in measuring the input impedance of each sub-part. The input impedance of the load $Z_{\text {load }}$ is measured as a function of the angular frequency $\omega$. The input impedance of the thermoacoustic core $Z_{t a c}$ is measured as a function of the angular frequency, of the heat power $Q$ supplied to the Nichrome wire, and of the peak amplitude $P$ of acoustic pressure measured with a third microphone mounted flush through the rigid plug facing the hot stack end (see Fig. 2). Note that this pressure amplitude $P$ can be controlled by the electrical power supplied to the impedance sensor. The interest in measuring the impedance of the two subparts is that the connection of each part amounts to matching their impedances, which can be expressed as

$$
Z_{\text {tac }}(\omega, Q, P) \neq-Z_{\text {load }}(\omega)
$$

where the minus sign in front of $Z_{\text {load }}$ comes from the choice of a direction for the acoustic volume velocity (oriented towards the thermoacoustic core). Eq. (8) is actually similar to the characteristic equation of the complete device ${ }^{18}$, and its solution provides a way to determine the possible steady-states associated with the autonomous oscillator considered. More precisely, if a given amount $Q$ of heat power is supplied to the TAC and if there exists a (real-valued) angular frequency $\omega_{\infty}$ as well as a (positive) pressure amplitude $P_{\infty}$ so that Eq. (8) is satisfied, then the solution $\left(\omega_{\infty}, P_{\infty}\right)$ corresponds to the frequency and the amplitude of a limit cycle potentially achieved by the system. Note, however, that Eq. (8) might have no solution (e.g. if the power supply is lower than a threshold value, or if the frequency range investigated does not include a natural frequency) or it might have several solutions (e.g. if the system is bistable, or if the frequency range investigated includes several natural frequencies).

If the measurements of $Z_{\text {tac }}(\omega, Q, P)$ and $Z_{\text {load }}(\omega)$ are performed within a given range of frequencies and pressure amplitudes, and for different values of the heat supply $Q$, then the post-processing of experimental data enables prediction of the steady-state potentially achieved by the device without any need to have information about the nonlinear processes responsible for the saturation of sound. The proof-of-concept of this procedure, which has been employed for several decades in the field of electronics ${ }^{28}$, is validated in the next section on the thermoacoustic engine described above. 


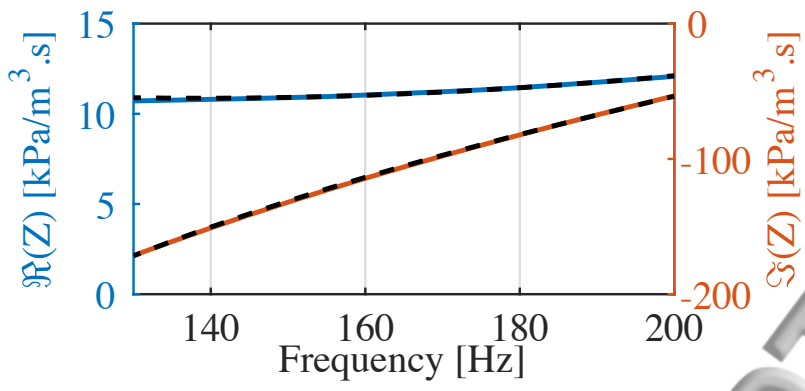

FIG. 3. Real part and imaginary part of the input impedance of the thermoacoustic core, $Z_{t a c}$, as functions of the frequency. The stack of length $L_{s}=4 \mathrm{~cm}$ is placed $22 \mathrm{~cm}$ away from the rigid plug, and the supplied heat power is switched off $(Q=0)$, the amplitude of acoustic pressure $P$ is around $100 \mathrm{~Pa}$, so that the measured impedance is a linear impedance which does not depend on the amplitude of acoustic forcing. Experiments (solid lines) are compared with the theoretical model (dashed lines). Note that the geometrical parameters of the stack are adjusted to match with the experimental data.

\section{EXPERIMENTAL RESULTS}

\section{A. Input impedance at low-amplitude, without heating}

The experimental results obtained for the input impedance of the thermoacoustic core are shown in Fig. 3, within the frequency range from $f=130 \mathrm{~Hz}$ up to $f=200 \mathrm{~Hz}$, and with the stack placed $22 \mathrm{~cm}$ away from the rigid plug (i.e. $\left.L_{t b t}=22 \mathrm{~cm}\right)$. These results are obtained without providing heat to the $\operatorname{TAC}(Q=0)$ and for a low-amplitude of acoustic forcing (around $100 \mathrm{~Pa}$ ), so the acoustically induced heat transport along the stack can considered as small, and the temperature distribution through the TAC can be considered as being uniform at room temperature. The input impedance is presented in terms of an acoustic resistance $\Re\left(Z_{t a c}\right)$ and an acoustic reactance $\Im\left(Z_{t a c}\right)$. As can be seen in Fig. 3 , the TAC has a negative reactance which grows with frequency: such a result is consistent with expectation because the wavelength is much larger than the length of the TAC within the frequency range investigated, so that the TAC mainly acts has a gas compliance $\left(i \Im\left(Z_{t a c}\right) \propto-i / \omega\right)$. The real part of the input impedance is always positive, which is also expected since sound amplification does not occur, and a positive power $\mathcal{W}_{i n}=\frac{1}{2} \Re\left(Z_{t a c}\right)\left|\tilde{u}_{i n}\right|^{2}$ must therefore be provided by the impedance sensor to sustain the acoustic field. 
PublishingAlthough the main scope of this study deals with experiments, it is interesting to compare the input impedances obtained experimentally to the ones obtained with a model based on Rott's theory $^{19}$. This model is presented in appendix A and it is used to calculate the input impedance as a function of the pressure amplitude $P$, the heat supply $Q$, and the angular frequency $\omega$. The model describes the thermoacoustic process within the stack, which is treated as a collection of parallel channels having a porosity $\phi$ and a mean channel radius $r_{0}$. The model also describes heat transport through the TAC, and it accounts both for the heat leaks to the lateral walls and for the thermoacoustic transport of heat. In Fig. 3, the results of the model are also presented (a linear version of the model with $Q=0$ is employed, since $Z_{t a c}$ does not depend on $P$ for low amplitudes). In the model the stack is treated as a collection of cylindrical pores, and the inner dimensions of the stack, namely the pore radius $r_{0}$ and the porosity $\phi$ have been adjusted to $r_{0}=0.57 \mathrm{~mm}$ and $\phi=0.75$ in such a way that a good agreement with the experimental results is obtained. It is worth noting that $\phi$ and $r_{0}$ are very close to the values specified by the manufacturer for 400 CPSI Celcor ${ }^{\circledR}$ and also to the values retrieved by Guedra et al. ${ }^{20}$ in a similar case. The theoretical value of the reactance matches well with the experimental one, and a small discrepancy is observed for the resistive part of $Z_{t a c}$ which is however not surprising regarding that the reactance is two orders of magnitudes higher than the resistance (the absolute error is actually almost the same for both $\Re\left(Z_{t a c}\right)$ and $\left.\Im\left(Z_{t a c}\right)\right)$.

\section{B. Input impedance at low-amplitude, with heating}

In Fig. 4, we present the real and imaginary parts of the input impedance $Z_{t a c}$ as functions of the heat supply $Q$ and for various frequencies of forcing, at low amplitude (heat transport by sound is therefore almost absent). The stack is placed at a distance of $22 \mathrm{~cm}$ from the rigid plug (i.e. $L_{t b t}=22 \mathrm{~cm}$ ). Here again, the results obtained experimentally are compared to the ones of the model. To that purpose, the geometrical parameters of the stack are kept the same $\left(r_{0}=0.57 \mathrm{~mm}, \phi=0.75\right)$ but another adjustment of the model parameters are made, which concerns the heat exchange coefficient $h_{\text {lat }}$ through the side walls along the stack and the remaining part of the TA core [see Appendix A, Eq. (A12)]. The determination of the heat exchange coefficients describing heat transport through the side walls is indeed difficult to obtain theoretically. Therefore, these coefficients are adjusted in the model in such a 


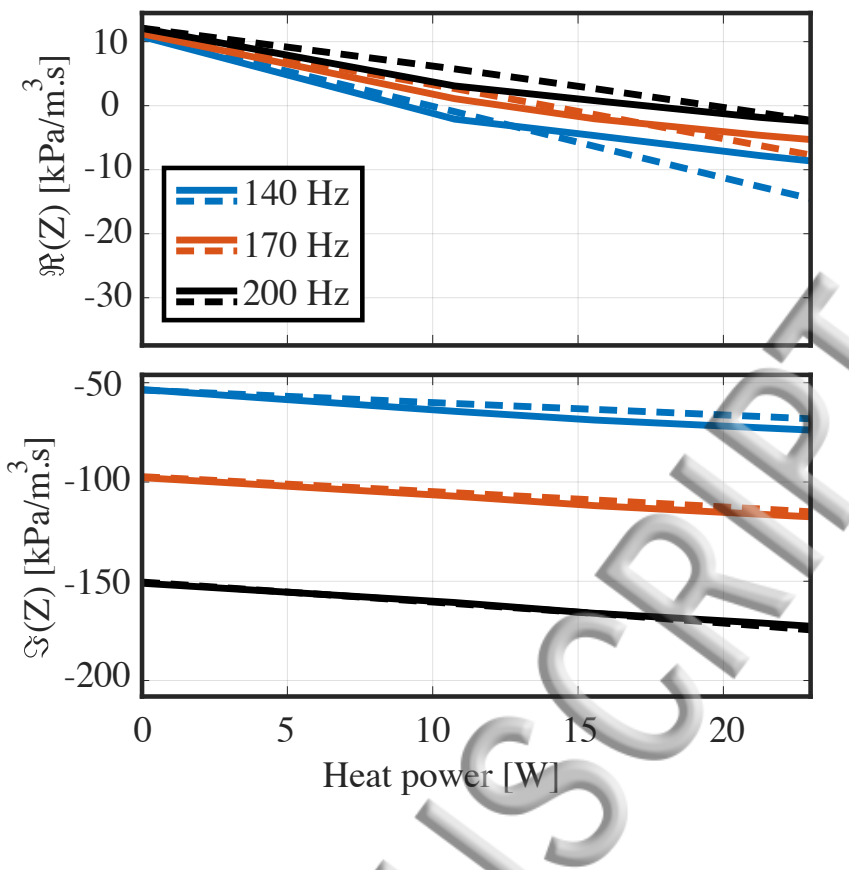

FIG. 4. (a) Real parts and (b) imaginary parts of the input impedance of a TAC as functions of the provided heat power for different acoustic driving frequencies. The stack of length $L_{s}=4 \mathrm{~cm}$ is placed at the distance $L_{t b t}=22 \mathrm{~cm}$ from the rigid plug of the TAC. Solid lines are obtained from the measurement, while the simulation results are represented with dashed lines. Note that in the model the coefficients $h_{\text {lat }}$ [see Appendix A, Eq. (A12)] describing heat leaks through lateral walls are adjusted so as to match with the experimental data.

way that the reactance of the TAC is close to the one observed in experiments. The values obtained once this adjustement procedure is achieved are a coefficient $h_{\text {lat }}=70 \mathrm{~W} / \mathrm{m}^{2} / \mathrm{K}$ for the description of lateral heat leaks through the side walls along the stack, and a coefficient $h_{\text {lat }}=10 \mathrm{~W} / \mathrm{m}^{2} / \mathrm{K}$ for the heat leaks through side walls along the remaining part of the thermoacoustic core. From the analysis of the results depicted in Fig. 4, one can clearly see that applying a temperature gradient to the TAC strongly impacts both the resistive and the reactive parts of the impedance. The variations of the reactance $\Im\left(Z_{t a c}\right)$ with heating can be explained by the fact that the propagation of sound is affected by temperature gradients (the speed of sound depends on temperature). More interestingly, the results clearly show, for both experiments and theory, that the supply of heat leads to a decrease of the resistance $\Re\left(Z_{t a c}\right)$ and even to a change of its sign, which means that above a critical value of heating, the TAC provides an acoustic gain instead of an acoustic attenuation. Note, however, that the occurrence of an acoustic gain (within a given frequency range) due to the thermoacoustic 
Publishiarg] lification process does not mean that self-sustained oscillations are generated, because the coupling of the TAC with the impedance sensor prevents this.

When comparing the results obtained experimentally with the ones obtained theoretically for the real part of $Z_{t a c}$, it appears that the model only reproduces qualitatively the experimental observation: in both cases the real part decreases with frequency so that acoustic attenuation becomes an acoustic gain, and in both cases the largest gain is obtained for the lowest frequency investigated $(140 \mathrm{~Hz})$. It is also observed, however, that the model does not reproduce perfectly the increase of the thermoacoustic gain as a function of the heating power. There are several reasons which may explain why such a difference is observed between experiments and theory, and we did not try here to reduce the discrepancy between the model and the experiments, which could have been done by trying to adjust parameters such as the effective thermal conductivity of the stack. Actually, the observed difference is not surprising when considering that the model describes heat transfer through the TAC in a very simplified way, which is based on a one-dimensional approximation (the temperature calculated by the model is a mean axial temperature averaged over the cross-sectional area of the duct). Therefore, it is important to note that in this study, the comparison with a model is performed with only the objective to facilitate the analysis of the experimental results obtained, and one could even argue that if a high-fidelity model of the TAC could be derived then the interest in characterizing the TAC as a black-box would become unnecessary.

Measurements are performed at low amplitudes of forcing, so that the input impedance of the thermoacoustic core can be considered as independent on the amplitude of acoustic pressure. If heat power is supplied to the TAC, a heterogeneous temperature distribution is produced but the impedance remains linear at low amplitudes of forcing (the impact of sound on heat transfer is low), and its real part exhibits amplification of sound when it reaches negative values (as shown in Fig. 4). However, at large amplitudes of forcing, the impedance of the TAC not only depends on the frequency and the heat supply $Q$, but also on the amplitude of the acoustic field which impacts the temperature distribution. This acoustically induced transport of heat is the effect which is investigated in the following, by means of impedance measurements. 


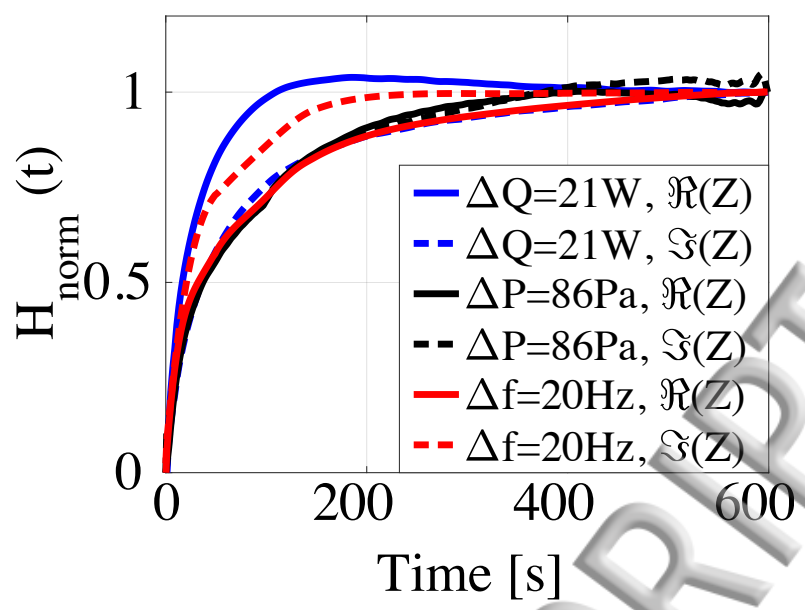

FIG. 5. Normalized step response $H_{\text {norm. }}(t)$, see Eq. (9), to an increment $\Delta Q$ of heat supply (blue lines), to an increment $\Delta f$ of frequency (red lines), or to an increment $\Delta P$ of pressure amplitude (black lines). The default values of $Q, P$ and $f$ are set to $Q_{0}=0 \mathrm{~W}, P_{0}=50 \mathrm{~Pa}$, and $f_{0}=150 \mathrm{~Hz}$, while the values of the increments are chosen as $\Delta Q=22 \mathrm{~W}, \Delta P=86 \mathrm{~Pa}$ and $\Delta f=20 \mathrm{~Hz}$.

\section{Input impedance as a function of heat supply and forcing amplitude.}

Prior to proceeding to an experimental mapping of $Z_{t a c}$ as a function of the frequency $f$, of the heat power $Q$, and of the peak amplitude $P$ of acoustic pressure measured by the microphone mounted flush in the rigid plug of the TAC, a first question needs to be addressed, which deals with the experimental protocol. Supplying heat to the device indeed leads to a transient regime of temperature variations, and the typical time of thermal equilibrium must be determined, since $Z_{t a c}$ must be measured once the temperature field has (almost) reached steady-state. Moreover, at large amplitudes of acoustic forcing and for a fixed value of heat power supply, any variation of frequency or any variation of the amplitude of sound also impacts the temperature distribution. Therefore, it is important on the one hand to make sure that the measurements of $Z_{t a c}(\omega, Q, P)$ are performed once the temperature field is constant, and on the other hand the shortest waiting time between two sets of measurements must be chosen so that the largest amount of data is obtained within a reasonable time. This question of a compromise to be found regarding the choice of a typical waiting time between two measurements is addressed by means of the experimental analysis of the step response of $Z_{t a c}$ to an increment of heating $\Delta Q$, of frequency $\Delta \omega$, or of pressure $\Delta P$. In Fig. 5 , the 
Publishipgogerimental results obtained for the transient response of $Z_{t a c}$ to an increment of acoustic pressure, of frequency, or of heat supply are presented. More precisely, the normalized step responses

$$
H_{\text {norm. }}(t)=\frac{F(t)-F(t=0)}{\operatorname{Max}(F(t))-F(t=0)}
$$

are presented as a function of time, where $F$ either refers to $\Re\left(Z_{t a c}\right)$ or to $\Im\left(Z_{t a c}\right)$. The step-responses to an increment in acoustic pressure (black lines), in heating (blue lines), or in frequency (red lines) are analyzed. From the results depicted in Fig. 5, one can conclude that, as a first approximation, both the real part and the imaginary of the input impedance $Z_{\text {tac }}$ present a step-response similar to the typical response of capacitor to a voltage increment, although the occurrence of an overshoot is visible for the step-response of $\Re\left(Z_{t a c}\right)$ on the solid blue curve around $\mathrm{t} \simeq 100 \mathrm{~s}$. Therefore, the results presented in Fig. 5 are used in the following as a guideline for the definition of an experimental protocol to measure $Z_{t a c}$. The protocol to get a complete map of $Z_{t a c}$ as a function of $P, Q$, and $\omega$ is chosen as follows:

1. the heat power supply is fixed to a value $Q_{\text {min }}$,

2. after a waiting time of $600 \mathrm{~s}$, the electrical power supplied to the loudspeaker is switched on, and forced oscillation with a frequency $f=f_{m i n}$, and with an amplitude $P=P_{\min }$ are provided to the TAC,

3. the input impedance $Z_{t a c}\left(\omega, P_{\min }, Q_{\min }\right)$ is measured as a function of frequency from $f=f_{\text {min }}$ up to $f=f_{\text {max }}$ with a sampling interval $\Delta f=5 \mathrm{~Hz}$, while a lag time of $300 \mathrm{~s}$ between two successive measurements is maintained,

4. an increment in voltage supplied to the impedance sensor is applied in such a way that the sound pressure amplitude rises by $\Delta P$ (with $\sim 100 \mathrm{~Pa}$ here), and the measurement of the frequency response function $Z_{t a c}\left(\omega, P_{\min }+\Delta P, Q_{\min }\right)$ is repeated (still with a lag time of $300 \mathrm{~s}$ between two successive measurements),

5. the latter operation is repeated to measure $Z_{t a c}\left(\omega, P, Q_{\min }\right)$ for pressure amplitudes ranging from $P_{\min }$ up to $P_{\max }$,

6. an increment $\Delta Q$ in heat power supply is provided (with $\Delta Q<5 \mathrm{~W}$ here), and the measurements are reproduced in accordance with the procedure above, to produce the 


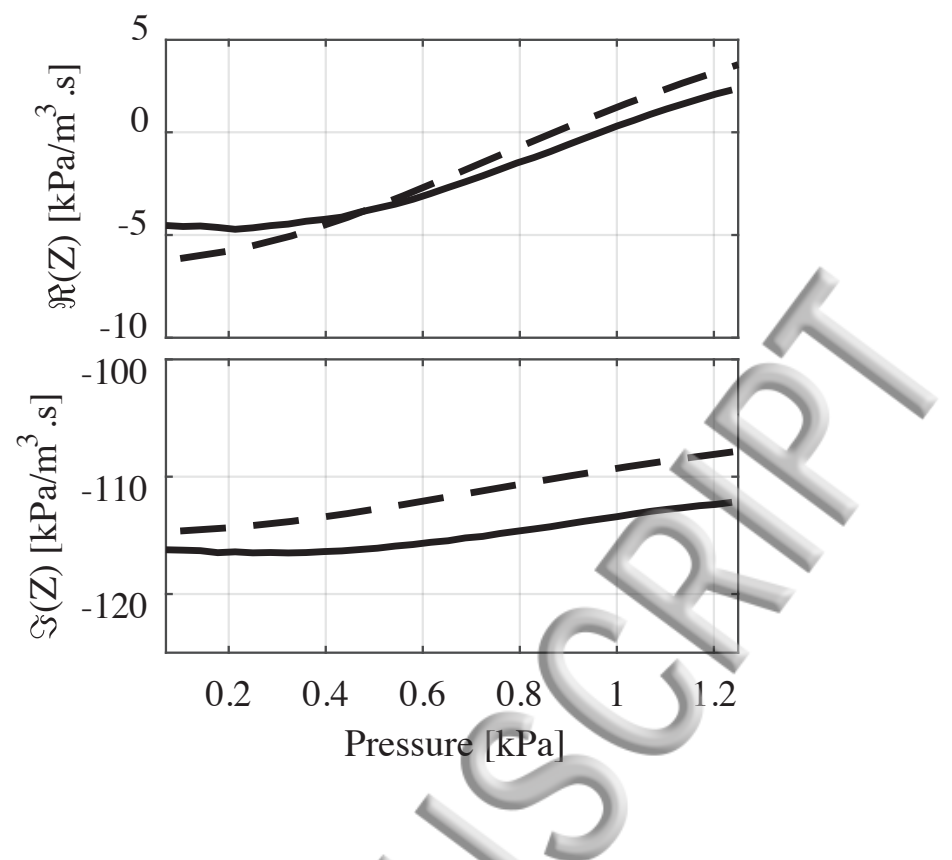

FIG. 6. Real part and imaginary part of the input impedance of a TAC as functions of the pressure amplitude $P$ for a provided heat power of $21 \mathrm{~W}$. The frequency of acoustic oscillations is fixed to $170 \mathrm{~Hz}$, and the stack is placed at a distance $L_{t b t}=22 \mathrm{~cm}$ from the plugged end. Together with the experimental results (solid line) a comparison of experimental data with the results of the theoretical model (dashed line) are presented.

complete map of $Z_{\text {tac }}(\omega, P, Q)$ with $Q$ ranging from $Q_{\min }$ up to $Q_{\max }$.

One set of measurements of $Z_{t a c}(\omega, P, Q)$ for a given position of the stack along the TAC gives rise to about $[15 \times 34 \times 4]$ data points.

In Fig. 6, a first set of measurements is presented, which corresponds to the variations of the real part of $Z_{t a c}$ as a function of the pressure amplitude $P$, for a frequency of $170 \mathrm{~Hz}$, and a heater power of $21 \mathrm{~W}$. The value of $Q$ is here such that at low amplitudes the real part of $Z_{t a c}$ is negative, which means that acoustic gain is provided by the thermoacoustic core. The results clearly show that an increase in acoustic pressure amplitude leads to an increase of $\Re\left(Z_{t a c}\right)$, as expected, and $\Re\left(Z_{t a c}\right)$ even vanishes around $P \approx 1 \mathrm{kPa}$. Such a result is expected, since the increase of acoustic pressure leads to an increase of the transport of heat by sound, which itself tends to reduce the temperature gradient (with subsequent diminution of thermoacoustic sound amplification). The associated results of the theoretical model are also presented in Fig. 6 using a dashed line: a good agreement is observed between measurement and theory (which might be explained by the fact that 
Publishing geometrical parameters of the stack as well as the heat exchange coefficients have been adjusted beforehand from the measurements at low amplitude, see subsection III A and III B).

\section{Prediction of the onset}

The results presented above show that the increase of the sound pressure level in the thermoacoustic core leads to a reduction of sound amplification, and therefore that a balance between the production and the dissipation of energy in the complete device of Fig. 2(a) should be achieved to determine an operating point corresponding to steady-state oscillations. The results of Fig. 6 also show that the impedance of the TAC remains almost constant for low amplitudes of forcing (typically less than $200 \mathrm{~Pa}$ ). Therefore, the results obtained for this linear regime where $Z_{\text {tac }}$ (almost) does not depend on $P$ can be used, at first, to determine the conditions for which the onset of the thermoacoustic instability occurs. As mentioned previously in Eq. (8), the connection of the TAC to its load is such that $Z_{\text {sum }}=Z_{\text {load }}+Z_{\text {tac }}$ must vanish. In the regime of low-amplitudes for which $Z_{t a c}$ is only a function of $Q$ and $\omega$, the onset condition therefore reduces to $Z_{\text {sum }}(\omega, Q)=0$ or to $-Z_{\text {load }}(\omega)=+Z_{\text {tac }}(\omega, Q)$. In Fig. $7(\mathrm{a}-\mathrm{b}-\mathrm{c}-\mathrm{d})$, the measured variations of the real parts and imaginary parts of $-Z_{\text {load }}(\omega)$ and $+Z_{t a c}(\omega, Q)$ as functions of the frequency are presented for various amounts $Q$ of heat supplied to the TAC. In this example, the impedance consists of a straight duct of length $L_{\text {load }}=20 \mathrm{~cm}$ terminated by an open end and the measured impedance $-Z_{\text {load }}(\omega)$ is presented with dashed lines, while the measured input impedances of the thermoacoustic core $+Z_{t a c}(\omega, Q)$ are presented with solid lines for heater powers ranging from $Q=10 \mathrm{~W}$ (soft grey lines) up to $Q=20 \mathrm{~W}$ (dark grey lines). Although the results presented in Fig. $7(\mathrm{a}-\mathrm{b}-\mathrm{c}-\mathrm{d})$ provide useful information, they do not provide an effective way to predict the onset of thermoacoustic oscillations: depending on the value of $Q$, it appears that the points of intersection of $-\Im\left(Z_{\text {load }}\right)$ with $+\Im\left(Z_{t a c}\right)$ are located within the frequency range $161 \mathrm{~Hz} \leq f \leq 166 \mathrm{~Hz}$ while the balance of the real parts occurs from $f=138 \mathrm{~Hz}$ (if $Q=10 \mathrm{~W}$ ) to $f=194 \mathrm{~Hz}$ (if $Q=20 \mathrm{~W}$ ). Since the objective is to find a value $Q_{\text {onset }}$ of $Q$ such that both the real part and the imaginary part of $Z_{\text {sum }}$ vanish for the same value $\omega_{\text {onset }}$ of the angular frequency, a more convenient way to present the results is the one in Fig. $7(\mathrm{e})$, where the functions $\Re\left(Z_{\text {sum }}\right)=0$ and $\Im\left(Z_{\text {sum }}\right)=0$ are presented as functions of 


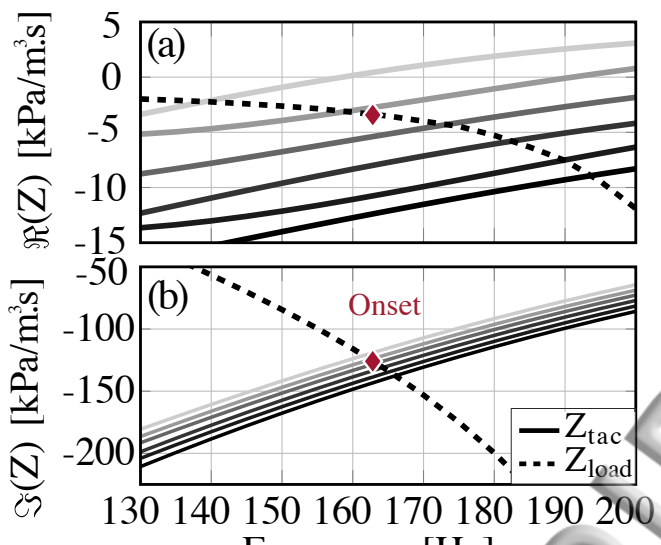

Frequency $[\mathrm{Hz}]$

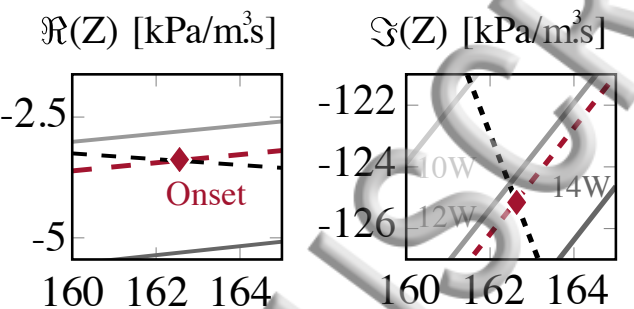

(c) Frequency $[\mathrm{Hz}](\mathrm{d})$ Frequency $[\mathrm{Hz}]$

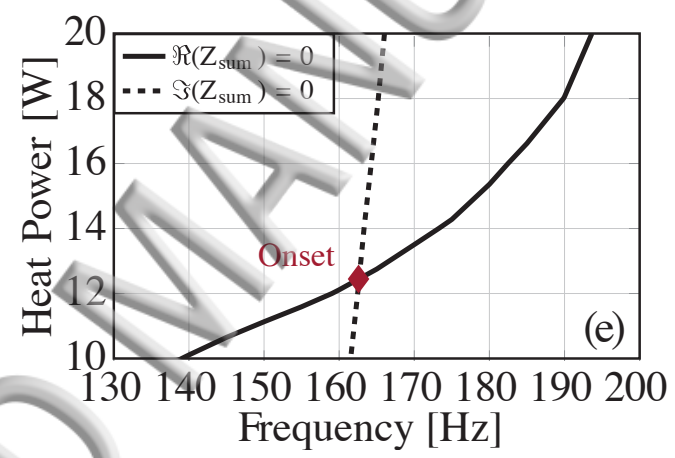

FIG. 7. (a-c) Real parts and (b-d) imaginary parts of $-Z_{\text {load }}$ and $+Z_{\text {tac }}$ measured as functions of the frequency, for a low amplitude of acoustic forcing (i.e. $P \leq 60 \mathrm{~Pa}$ ) and for various amounts of heat supplied to the TAC ranging from $Q=10 \mathrm{~W}$ (soft grey lines) up to $Q=20 \mathrm{~W}$ (dark grey lines). (e) Plot of the functions $\Re\left(Z_{\text {sum }}\right)=0$ and $\Im\left(Z_{\text {sum }}\right)=0$ as functions of both the frequency and the heater power. These functions are obtained from a contour plot of experimental data, where the total impedance $Z_{\text {sum }}$ is defined as $Z_{\text {sum }}=Z_{\text {load }}+Z_{\text {tac }}$.

the frequency and the heater power. These functions are obtained from the post-processing of experimental data using the contour plot tool in the Matlab ${ }^{\circledR}$ environment. The results of Fig. 7(e) show that the onset condition occurs at the intersection of the two curves, i.e. at a frequency $f_{\text {onset }} \approx 162.5 \mathrm{~Hz}$ and a heater supply $Q_{\text {onset }} \approx 12.5 \mathrm{~W}$. The same, but less visible conclusion can be drawn from the analysis of Fig. 7(a-b-c-d), and this prediction has 


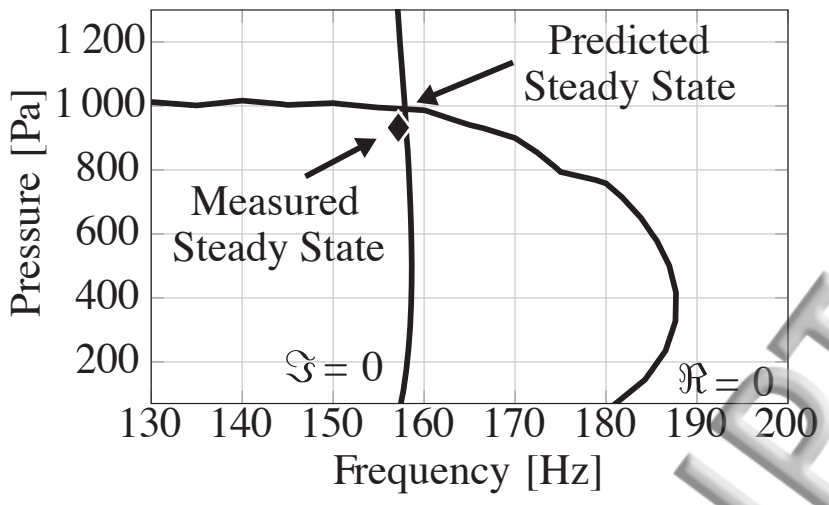

FIG. 8. Plot of the curves $\Re\left(Z_{\text {sum }}\right)=0$ and $\Im\left(Z_{\text {sum }}\right)=0$ as functions of the frequency and of the amplitude of acoustic pressure for provided heat power of $Q=22.5 \mathrm{~W}$. The curves cross each other at $f_{\infty}=157.5 \mathrm{~Hz}$, and $P_{\infty}=990 \mathrm{~Pa}$, which corresponds to the impedance matching condition. On the same plot the measured limit cycle with $f=157.1 \mathrm{~Hz}$ and $P=938 \mathrm{~Pa}$ is represented using a diamond marker.

also been confirmed by performing the experiment in the complete thermoacoustic device. The mode of representation of the measured data adopted in Fig. 7(e) is the one retained in the following, where the extension to large amplitudes of forcing is investigated to predict the frequency and the amplitude of steady-state oscillations.

\section{E. Prediction of steady-state behavior}

The impedance matching condition of Eq. (8) remains applicable for the post-processing of experimental data obtained for large amplitudes of acoustic forcing, and a graphical analysis similar to the one of Fig. 8 can be used to predict the steady-state angular frequency $\omega_{\infty}$ as well as the associated peak amplitude of acoustic pressure $P_{\infty}$ for any value of the heat power $Q>Q_{\text {onset }}$ supplied to the TAC. This analysis is performed in the case of a load consisting of a duct of length $L_{\text {load }}=20 \mathrm{~cm}$ terminated with an open end, while the heater power is fixed to $Q=22.5 \mathrm{~W}$. The results obtained after post-processing of the measured impedances $Z_{\text {load }}(\omega)$ and $Z_{\text {tac }}(\omega, P, Q)$ are presented in Fig. 8. According to these measurements, the device should give rise to a steady-state with a frequency $f_{\infty} \approx 157.5 \mathrm{~Hz}$ and an amplitude $P_{\infty} \approx 990 \mathrm{~Pa}$. Note that the frequency of the predicted self-sustained oscillations remains, as expected, close to the first resonance frequency $f=c_{0} /(4 L) \approx 159 \mathrm{~Hz}$ 

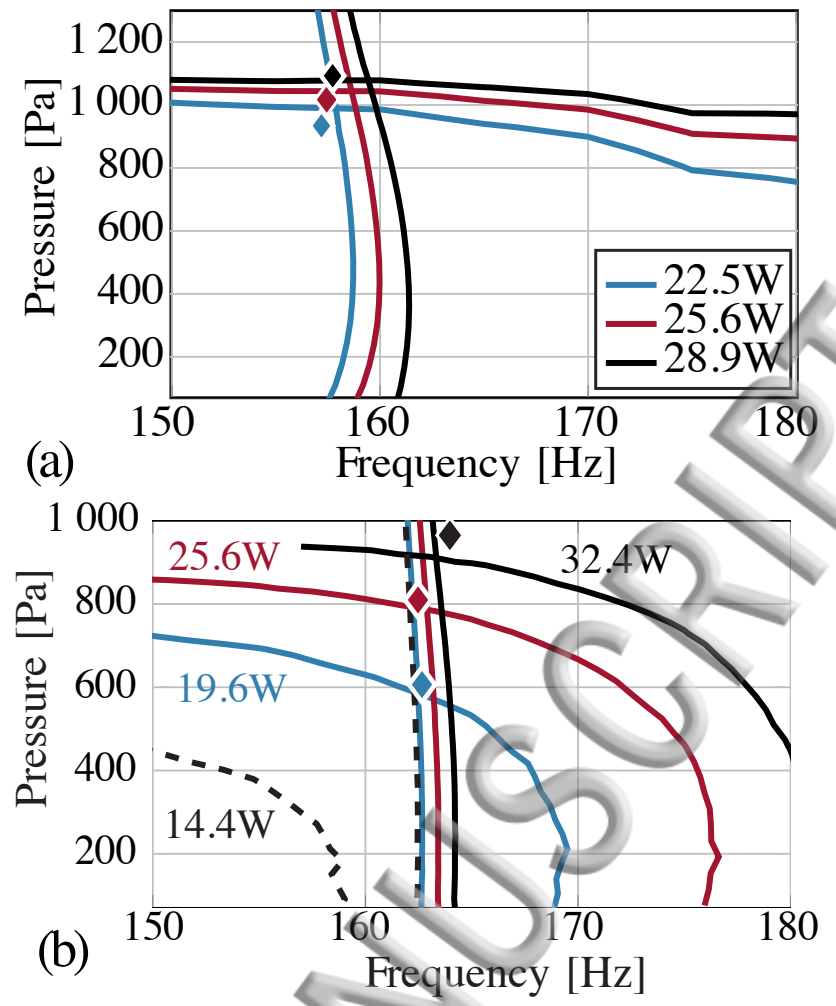

FIG. 9. Steady-state prediction from the measurements of impedances $Z_{\text {load }}(\omega)$ and $Z_{\text {tac }}(\omega, P, Q)$ and comparison with the steady-state amplitudes observed in experiments (diamond markers). (a) Case of a quarter-wavelength thermoacoustic prime-mover, where the load is a straight-duct of length $L_{\text {load }}=20 \mathrm{~cm}$ terminated by an open end, and for three different values of the heat power supply. (b) Case of a half-wavelength thermoacoustic prime-mover, where the load is a straightduct of length $L_{\text {load }}=72.9 \mathrm{~cm}$ terminated by rigid plug, and for four different values of the heat power supply.

of a quarter wavelength resonator of length $L=L_{\text {load }}+L_{\text {tac }}$ filled with air at 1 bar and at room temperature $\left(c_{0} \approx 344 \mathrm{~m} / \mathrm{s}\right)$. The occurrence of self-sustained acoustic oscillations has been checked afterwards, by connecting the load to the TAC, and applying the same heater power to the device. The amplitude of the acoustic pressure is $P_{\infty}=938 \mathrm{~Pa}$ at a frequency $f_{\infty}=157.1 \mathrm{~Hz}$, as shown in Fig. 8 with a diamond marker. The predicted steady-state pressure amplitude and onset threshold is therefore close to the one observed in experiments.

Additional proof of concept experiments have been performed, where the amplitude of heating, the position of the stack, or the load have been changed to check that the predictions of steady-state remain close to the experimental observations. In Figs. 9, the impact of the 
Publishihgoting power supplied to the TAC is investigated for both cases of a load terminated with an open end (a), or a load terminated with a rigid plug (b). The results show that the predictions of acoustic pressure amplitude and frequency remain close to the ones observed in experiments. Moreover, it is worth noting that the absence of thermoacoustic oscillations can also be predicted, and confirmed by experiments: this is indeed the case in Fig. 9(b) for $Q=14.4 \mathrm{~W}$, where the analysis does not lead to a possibility for impedance matching, just because the heat power supplied is too low (the absence of thermoacoustic oscillations was also confirmed by experiments).

The discrepancies observed in Figs. 9 (a) and (b) between the predicted steady-states and the ones actually observed can be explained by several sources of errors. The most important source of errors is probably the one due to the experimental procedure used to produce the mapping of $Z_{i n}$ as a function of $Q, \omega$ and $P$. The thermal inertia of the system is indeed such that the measurements of $Z_{i n}$ had to be made for a limited number of frequencies, of heat power supply and of amplitudes of forcing, regarding that a latency time of $300 \mathrm{~s}$ was required between two successive measurements. The quality of the sensor's calibration is also an important source of error, which may impact significantly the predicted steady-states : the determination of the admittances $Y_{1,2}$ as functions of $\omega$ has to be made carefully through the two loads calibration procedure. We could check, however, that the determination of the admittances $Y_{1,2}$ as functions of the amplitude of forcing is a less critical aspect of the calibration, since removing this nonlinear calibration in the post-processing of experimental data/leads to small variations of the predicted steady-states (the variations of $P_{\infty}$ were typically less than $\left.1 \%\right)$. It is also worth pointing out that even if the quality of measurements should be further improved, there would still be some possible discrepancies between the predicted steady-states and the ones actually observed, because some sources of sound saturation may depend on whether the TAC is connected to the impedance sensor or to an acoustical load : this might be the case, for instance, if acoustic streaming or free convection should contribute significantly to the temperature distribution within the TAC.

\section{CONCLUSION}

In this study, we have presented an experimental approach based on the measurements of the input impedance of a thermoacoustic core at large amplitudes of acoustic forcing, from 
Publishiwgich it is possible to predict the amplitude and the frequency of steady-state thermoacoustic oscillations once this thermoacoustic core is connected to a load. The proof of concept of this approach has been provided successfully for various configurations of engines, even though small discrepancies between the experimental results and the prediction have been observed.

The new method described in this paper could find useful applications for the development of high power thermoacoustic engines, as it provides a way to optimize the coupling between a thermoacoustic core and its load. It is indeed worth remembering that the prediction of the performances of thermoacoustic engines are often an overestimate of the performances actually reached once the engine is operational. This is due to the fact that the design tools have some known limitations related to the difficulty of describing accurately the operation of such elements like the stack/regenerator or the heat exchangers at large amplitudes of acoustic oscillations. Therefore, the measurement of the input impedance provides means to characterize all of these elements as a whole at an operational amplitude and the optimization process then comes down to determining an appropriate load that maximizes the overall efficiency or the useful power.

The works described in this study also appeal for several perspectives which are described below. The characterization of the load in terms of a (linear) input impedance could also be extended to large amplitudes. This is not necessary for the loads employed in this study (as far as the impact of nonlinear propagation is negligible), but it might be important for other loads like electromagnetic alternators or bidirectional turbines employed for the conversion of acoustical power into electrical power, or for other loads like thermoacoustic heat-pumps (supplied by a thermoacoustic engine). Another extension of this study would be to measure the transfer matrix of the thermoacoustic core for large amplitudes of acoustic oscillations: measuring an input impedance indeed has some limitations because one has to connect the thermoacoustic core (a two-port network) with a rear load (a rigid plug was used in this study). It would therefore be interesting to develop an experimental set-up enabling to measure the four elements of a transfer matrix (instead of an input impedance) as functions of the pressure and the velocity amplitudes at both ends of the thermoacoustic core: this would avoid consideration of a rear load (as done in this study), or from placing the thermoacoustic core in a loop (as done for instance in ref. ${ }^{23}$ ). Finally, it would be interesting for future works to address the question of (prediction of) the stability of limit cycles, because several experimental works have shown that the onset of thermoacoustic 
Publishipgegillations sometimes gives rise to complicated dynamics involving multiple stability ${ }^{20}$ and hysteretic behaviors ${ }^{29}$.

\section{ACKNOWLEDGEMENTS}

This research was funded by the program 'Le Mans Acoustique HUB'.

\section{Appendix A: Computation of $Z_{t a c}$}

In this appendix, the derivation of the equations used to compute the input impedance $Z_{t a c}(\omega, Q, P)$ are presented. For the sake of conciseness, and because further details can be found in ref. ${ }^{20}$ or elsewhere ${ }^{2,3,19,30}$, only the main principles of the calculation are provided here. The system considered is the thermoacoustic core presented in Fig. 10, which itself is composed of three parts, namely the front duct, the stack, and the thermal buffer tube (TBT). The calculation of $Z_{t a c}(\omega, Q, P)$ is based on the linear thermoacoustic theory ${ }^{3}$. The stack has a porosity $\phi$ and is treated as a collection of adjacent parrallel channels having an effective pore radius $r_{0}$. Linear propagation of plane pressure waves is assumed and the boundary layer approximation is also used to write the continuity, the momentum, and the energy equations averaged over the cross-sectional area of each channel. The front duct and the thermal buffer tube are described with the same equations, but they consist of a unique channel with inner radius $R_{\text {tac }}$ and a porosity of 1 . Therefore, the lossy propagation of plane waves in each part of the TAC is written $\mathrm{as}^{2}$

$$
\begin{aligned}
\frac{d \tilde{p}}{d x} & =-\frac{i \omega \rho_{0}}{\phi S} \frac{1}{1-f_{\nu}} \tilde{u} \\
\frac{d \tilde{u}}{d x} & =-\frac{i \omega \phi S}{\gamma P_{0}}\left[1+(\gamma-1) f_{\kappa}\right] \tilde{p} \\
& +\frac{f_{\kappa}-f_{\nu}}{\left(1-f_{\nu}\right)(1-\sigma)} \frac{d T_{0}}{d x} \frac{1}{T_{0}} \tilde{u}
\end{aligned}
$$

where $\tilde{p}$ and $\tilde{u}$ respectively stand for the complex amplitudes of acoustic pressure and volume velocity, $\rho_{0}(x), P_{0}$, and $T_{0}(x)$ stand for the mean (time-averaged) density, the mean pressure and the mean axial temperature, respectively, $\gamma$ is the specific heat ratio of the fluid, $S=$ $\pi R_{t a c}^{2}$ corresponds to the cross-sectional area of the TAC, $\sigma=\nu_{\infty} / \kappa_{\infty}$ is the Prandtl number of the fluid $\left(\nu_{\infty}\right.$ and $\kappa_{\infty}$ are the kinematic viscosity and the thermal diffusivity of the fluid 


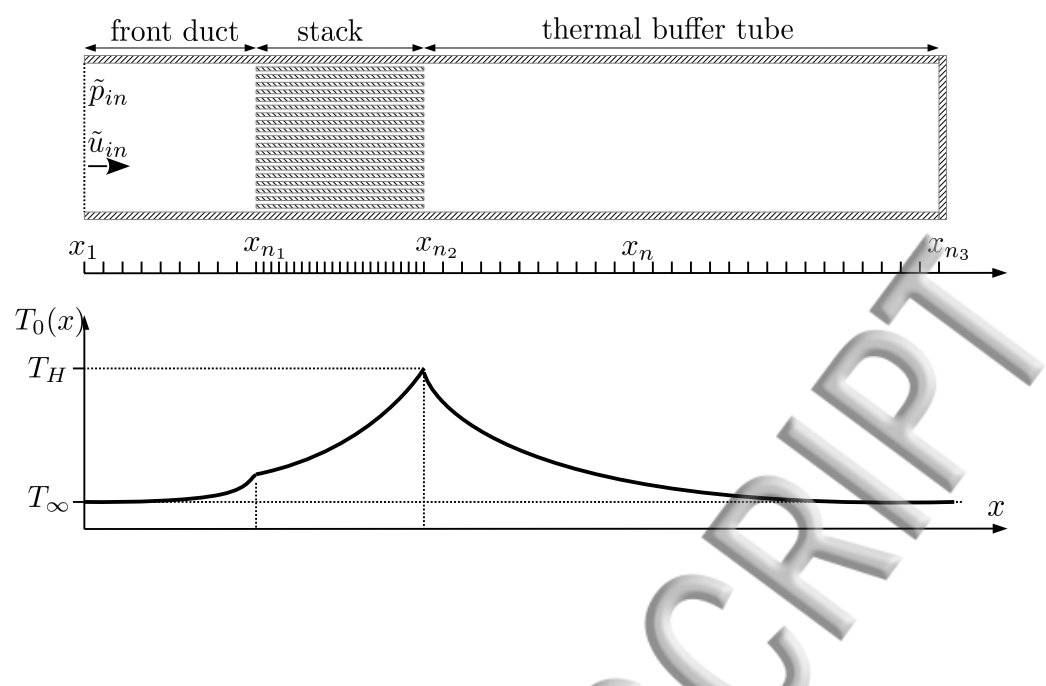

FIG. 10. Geometry of the problem considered for the computation of the input impedance of the thermoacoustic core $Z_{t a c}=\tilde{p}_{i n} / \tilde{u}_{i n}$.

at room temperature $\left.T_{\infty}\right)$, and $\phi$ is the porosity of the element $(\phi<1$ in the stack, and $\phi=1$ elsewhere). In Eqs. (A1)-(A2), the thermoacoustic process is described through the thermoviscous functions $f_{\kappa}$ and $f_{\nu}$. These functions depend on the shape and on the dimensions of the channel. Introducing the characteristic time $\tau=r_{0}^{2} / 2 \kappa_{\infty}$, the function $f_{\kappa}$ (evaluated at room temperature $T_{\infty}$ ) is given $b^{2}$ :

$$
f_{\kappa}=\frac{2}{(1-i) \sqrt{\omega \tau}} \frac{J_{1}[(1-i) \sqrt{\omega \tau}]}{J_{0}[(1-i) \sqrt{\omega \tau}]}
$$

for each cylindrical channel of radius $r_{0}$, where $J_{m}$ is the Bessel function of order $m$. The function $f_{\nu}$ is easily obtained from $f_{\kappa}$, just by replacing $\tau$ with $\tau / \sigma$ in the equation above.

A discrete version of these equations is used to solve the problem under consideration, and accordingly with the notations introduced in Fig. 10, the acoustic propagation through a short portion of length $\Delta x_{n}=x_{n+1}-x_{n}$ (with $\Delta x_{n}$ much shorter than the wavelength $2 \pi c / \omega)$, is described from Eqs. (A1)-(A2) by means of a forward Euler finite-difference scheme (i.e. setting $(d \xi / d x) \approx\left(\xi_{n+1}-\xi_{n}\right) / \Delta x_{n}$ where $\xi$ either stands for $\tilde{p}, \tilde{u}$ or $T$ ) and is written as follows: 


$$
\begin{aligned}
\tilde{p}_{n+1} & =\tilde{p}_{n}-\frac{i \omega \rho_{n} \Delta x_{n}}{\phi S} \frac{1}{1-f_{\nu_{n}}} \tilde{u}_{n}, \\
\tilde{u}_{n+1} & =-\frac{i \omega \phi S \Delta x_{n}}{\gamma P_{0}}\left[1+(\gamma-1) f_{\kappa_{n}}\right] \tilde{p}_{n} \\
& +\left[1+\frac{f_{\kappa_{n}}-f_{\nu_{n}}}{\left(1-f_{\nu_{n}}\right)(1-\sigma)} \frac{T_{n+1}-T_{n}}{T_{n}}\right] \tilde{u}_{n},
\end{aligned}
$$

where $\tilde{p}_{n}, \tilde{u}_{n}$ and $T_{n}$ stand for the pressure, the volume velocity, and the mean temperature at position $x=x_{n}$, where $\rho_{n}=\rho_{\infty} T_{\infty} / T_{n}$ stands for the fluid density at temperature $T_{n}$, and where the variations of the viscothermal functions $f_{\nu_{n}, \kappa_{n}}=f_{\nu, \kappa}\left(\omega, T_{n}\right)$ with temperature, due to the variations of $\nu$ and $\kappa$ with $T_{n}$, are taken into account using the empirical laws $\nu_{n}=\nu_{\infty}\left(T_{n} / T_{\infty}\right)^{1+\beta}$ and $\kappa_{n}=\kappa_{\infty}\left(T_{n} / T_{\infty}\right)^{1+\beta}$ (with $\beta=0.77$ for air $^{31}$ ). If the temperature distribution is assigned and known for any position $x_{n}$ along the TAC, Eqs. (A4)-(A5) can be solved to compute the spatial distribution of the acoustic field, and therefore to calculate the input impedance of the TAC, $Z_{i n}=\tilde{p}_{1} / \tilde{u}_{1}$. However, the temperature distribution is not assigned here, and a heat power $Q$ is supplied instead, at position $x=x_{n_{2}}$. This leads to the establishment of a temperature distribution which is controled by heat conduction and heat leaks through lateral walls, and by the thermoacoustic transport of heat through the stack (which itself depends on the amplitude and the spatial distribution of the acoustic field).

The equations describing the steady-state temperature distribution through the stack are therefore written as

$$
\begin{aligned}
0 & =\frac{T_{n-1}-T_{n}}{R_{n-1}^{t h}}-\frac{T_{n}-T_{n+1}}{R_{n}^{t h}}-\frac{T_{n}-T_{\infty}}{R_{l a t}^{t h}} \\
& +Q_{2}\left(\tilde{p}_{n}, \tilde{u}_{n}\right)-Q_{2}\left(\tilde{p}_{n-1}, \tilde{u}_{n-1}\right)
\end{aligned}
$$

where $R_{n}^{t h}$ stands for the thermal resistance which accounts for both axial conduction and acoustically enhanced thermal conduction, where $R_{\text {lat }}^{\text {th }}$ refers to the thermal resistance associated to the transverse heat transport towards lateral walls, and where $Q_{2}$ stands for the component of thermoacoustic heat flux which does not depend on the axial temperature gradient. Each of these terms is written as follows :

$$
\begin{array}{r}
R_{n}^{t h}=\frac{\Delta x}{\left(\lambda_{s}+\Gamma_{\lambda_{n}}\left|\tilde{u}_{n}\right|^{2}\right) S} \\
Q_{2}\left(\tilde{p}_{n}, \tilde{u}_{n}\right)=\frac{1}{2 \phi} \Re\left(\tilde{g}_{n} \tilde{p}_{n} \tilde{u}_{n}^{*}\right)
\end{array}
$$


Publishiwgere $\lambda_{s}$ refers to the thermal conductivity of the stack, which is estimated as $\lambda_{s}=0.6$ $\mathrm{W} / \mathrm{m} / \mathrm{K}$ accordingly with previous works ${ }^{20}$. The thermoacoustic functions $\tilde{g}_{n}$ and $\Gamma_{\lambda_{n}}$ are defined as in $\operatorname{ref}^{30}$

$$
\begin{array}{r}
\tilde{g}_{n}=\frac{\sigma f_{\nu_{n}}^{*}-f_{\kappa_{n}}}{\left|1-f_{\nu_{n}}\right|^{2}}, \\
\Gamma_{\lambda_{n}}=\frac{1}{(\phi S)^{2}} \frac{\rho_{n} C_{p}}{2 \omega\left(1-\sigma^{2}\right)} \Im\left(\tilde{g}_{D_{n}}\right) .
\end{array}
$$

where the notation $f^{*}$ refers to the conjugate of the complex number $f$, and where $\tilde{g}_{D_{n}}$ is defined as

$$
\tilde{g}_{D_{n}}=\frac{f_{\nu_{n}}^{*}-f_{\kappa_{n}}}{(1+\sigma)\left(1-f_{\nu_{n}}^{*}\right)}
$$

An order of magnitude estimate of the thermal resistance

$$
R_{\text {lat }}^{t h}=\frac{1}{2 \pi R_{\text {tac }} \Delta x_{n} h_{\text {lat }}}
$$

can be obtained from the estimate of the heat exchange coefficient $h_{\text {lat }}$ which itself depends on the geometry and the thermophysical properties of each element (e.g. see ${ }^{20,33}$ ). In this study, the heat exchange coefficient is adjusted so that the model fits with experiments (as described in Sect. III), leading to $h_{\text {lat }}=70 \mathrm{~W} / \mathrm{m}^{2} / \mathrm{K}$, if $x_{n_{1}} \leq x \leq x_{n_{2}}$ and to $h_{\text {lat }}=$ $10 \mathrm{~W} / \mathrm{m}^{2} / \mathrm{K}$, if $0 \leq x \leq x_{n_{1}}$ or $x_{n_{2}} \leq x \leq x_{n_{3}}$. The same equations as above are also used to describe heat transport through the front duct and the thermal buffer tube, except that the values of thermo-physical parameters (e.g. thermal conductivity, density) are changed accordingly with the change of properties of the medium, and the thermoacoustic transport of heat is neglected. The boundary conditions at the interfaces between each part of the TAC are written to ensure the continuity of the temperature and of the axial heat flux at position $x=x_{n_{1}}$, while the effect of heating at position $x=x_{n_{2}}$ is written as

$$
Q=\frac{T_{N_{2}}-T_{N_{2}-1}}{R_{N_{2}-1}^{t h}}+\frac{T_{N_{2}+1}-T_{N_{2}}}{R_{N_{2}}^{t h}}
$$

where $R_{n_{2}}^{t h}=\frac{\Delta x_{n}}{\lambda_{n_{2}} S}$ is thermal resistance associated to the axial heat conduction through the thermal buffer tube, and where $\lambda_{n_{2}}$ refers to the thermal conductivity of the fluid at position $x=x_{n_{2}}$, which itself depends on temperature through the empirical relation $\lambda_{n_{2}}=$ $\lambda_{\infty}\left(T_{n_{2}} / T_{\infty}\right)^{\beta}$, and where $\lambda_{\infty}=2.26 \mathrm{~W} / \mathrm{m} / \mathrm{K}$ is the thermal conductivity of air at room temperature $T_{\infty}$.

The equations describing the steady-state temperature field as a function of the heat supply and of both classical and acoustically induced thermal diffusion are coupled to the 
Publishimgrations describing the propagation of acoustic waves through the TAC. This leads to a nonlinear system of equations which is solved numerically. If the angular frequency of acoustic oscillations and the amplitude $P$ of acoustic pressure at position $x=x_{n_{3}}$ are imposed, then it is therefore possible to calculate the input impedance of the TAC, $Z_{t a c}(\omega, P, Q)$, for any value of the heat power $Q$ supplied to the TAC.

\section{REFERENCES}

${ }^{1}$ S. Temkin. Elements of Acoustics. Wiley, 1981.

${ }^{2}$ G. W. Swift. Thermoacoustics: A Unifying Perspective for Some Engines and Refrigerators. ASA Press / Springer, 2nd ed. edition, 2018.

${ }^{3}$ G. W. Swift. Thermoacoustic engines. J. Acoust. Soc. Am., 84(4):1145-1180, 1988.

${ }^{4}$ S. Backhaus and G. W. Swift. A thermoacoustic-Stirling heat engine: Detailed study. J. Acoust. Soc. Am., 107(6):3148-3166, 2000.

${ }^{5}$ M. E. H. Tijani and S. Spoelstra. A high performance thermoacoustic engine. J. Appl. Phys., 110(9):93519, 2011.

${ }^{6}$ T. Bi, Z. Wu, L. Zhang, G. Yu, E. Luo, and W. Dai. Development of a 5kW traveling-wave thermoacoustic electric generator. Appl. Energy, 185:1355 - 1361, 2017.

${ }^{7}$ S. L. Garrett, J. A. Smith, R. W.M. Smith, B. J. Heidrich, and M.D. Heibel. Fissionpowered in-core thermoacoustic sensor. Appl. Phys. Lett., 108(14), 2016.

${ }^{8}$ K. Wang, S. R. Sanders, S. Dubey, F. H. Choo, and F. Duan. Stirling cycle engines for recovering low and moderate temperature heat: A review. Renew. Sustain. Energy Rev., 62(Supplement C):89 - 108, 2016.

${ }^{9}$ L. A. Wilen. Dynamic measurements of the thermal dissipation function of reticulated vitreous carbon. J. Acoust. Soc. Am., 109(1):179-184, 2001.

${ }^{10}$ M. Guédra, F. C. Bannwart, G. Penelet, and P. Lotton. Parameter estimation for the characterization of thermoacoustic stacks and regenerators. Appl. Therm. Eng., 2015.

${ }^{11} \mathrm{P}$. Blanc-Benon, E. Besnoin, and O. Knio. Experimental and computational visualization of the flow field in a thermoacoustic stack. Comptes Rendus Mécanique, 331(1):17 - 24, 2003.

${ }^{12}$ A. Berson, G. Poignand, P. Blanc-Benon, and G. Comte-Bellot. Nonlinear temperature field near the stack ends of a standing-wave thermoacoustic refrigerator. Int. J. Heat Mass 
${ }^{13}$ G. Penelet, M. Leclercq, T. Wassereau, and P. Picart. Measurement of density fluctuations using digital holographic interferometry in a standing wave thermoacoustic oscillator. Exp. Therm. Fluid Sci., 70:176-184, 2016.

${ }^{14}$ S. Boluriaan and P. J. Morris. Acoustic Streaming: From Rayleigh to Today. Int. J. Aeroacoustics, 2(3):255-292, 2003.

${ }^{15}$ C. Desjouy, G. Penelet, P. Lotton, and J. Blondeau. Measurement of acoustic streaming in a closed-loop traveling wave resonator using laser Doppler velocimetry. J. Acoust. Soc. Am., 126(5):2176-2183, 2009.

${ }^{16}$ C. Olivier, G. Penelet, G. Poignand, J. Gilbert, and P. Lotton. Weakly nonlinear propagation in thermoacoustic engines: A numerical study of higher harmonics generation up to the appearance of shock waves. Acta Acust. united with Acust., 101:941-949, 2015.

${ }^{17}$ J. P. Clark, W. C. Ward, and G. W. Swift. Design environment for low amplitude thermoacoustic energy conversion (DeltaEC). J. Acoust. Soc. Am., 122(5):3014, 2007.

${ }^{18} \mathrm{M}$. Guédra and G. Penelet. On the Use of a Complex Frequency for the Description of Thermoacoustic Engines. Acta Acust. united with Acust., 98:232-241, apr 2012.

${ }^{19}$ N. Rott. Thermoacoustics. Adv. Appl. Mech., 20:135-175, 1980.

${ }^{20} \mathrm{M}$. Guedra, G. Penelet, and P. Lotton. Experimental and theoretical study of the dynamics of self-sustained oscillations in a standing wave thermoacoustic engine. J. Appl. Phys., 115(024504), 2014.

${ }^{21}$ M. Guedra, G. Penelef, P. Lotton, and J.-P. Dalmont. Theoretical prediction of the onset of thermoacoustic instability from the experimental transfer matrix of a thermoacoustic core. J. Acoust. Soc. Am., 130:145-152, 2011.

${ }^{22}$ F. C. Bannwart, G. Penelet, P. Lotton, and J.-P. Dalmont. Measurements of the impedance matrix of a thermoacoustic core: Applications to the design of thermoacoustic engines. $J$. Acoust. Soc. Am., 133:2650-2660, 2013.

${ }^{23}$ H. Hatori, T. Biwa, and T. Yazaki. How to build a loaded thermoacoustic engine. J. Appl. Phys., 111(7):74905, 2012.

${ }^{24}$ T. Holzinger, T. Emmert, and W. Polifke. Optimizing thermoacoustic regenerators for maximum amplification of acoustic power. J. Acoust. Soc. Am., 136(5):2432-2440, 2014.

${ }^{25}$ J.-P. Dalmont. Acoustic Impedance Measurement, Part I: A Review. J. Sound Vib., 243(3):427-439, 2001. 
Publishiîfg. P. Dalmont. Acoustic impedance measurement, Part II: A new calibration method. $J$. Sound Vib., 243(3):441-459, 2001.

${ }^{27}$ W. Chester. Resonant oscillations in closed tubes. J. Fluid Mech., 18(1):44-64, 1964.

${ }^{28}$ A. Gelb and W.E. Van Der Velde. Multiplie-input Describing Function and Nonlinear System Design. Mc Graw-Hill Electronic Sciences Series, 1968.

${ }^{29}$ G. Poignand, C. Olivier, G. Penelet, and P. Lotton. Hysteretic behavior induced by an electroacoustic feedback loop in a thermo-acousto-electric generator. Appl. Acoust., 105:110-115, 2016.

${ }^{30}$ A. Tominaga. Thermodynamic aspects of thermoacoustic theory. Cryogenics (Guildf)., 35(7):427-440, 1995.

${ }^{31}$ H. Bailliet, V. Gusev, R. Raspet, and R. A. Hiller. Acoustic streaming in closed thermoacoustic devices. J. Acoust. Soc. Am., 110(4):1808-1821, 2001.

${ }^{32}$ M.N. Özisik. Heat Transfer : A Basic Approach. Vol. 1 edition, 1985.

${ }^{33}$ G. Penelet, V. Gusev, P. Lotton, and M. Bruneau. Experimental and theoretical study of processes leading to steady-state sound in annular thermoacoustic engines. Phys. Rev. E - Stat. Nonlinear, Soft Matter Phys., 72(1), 2005. 


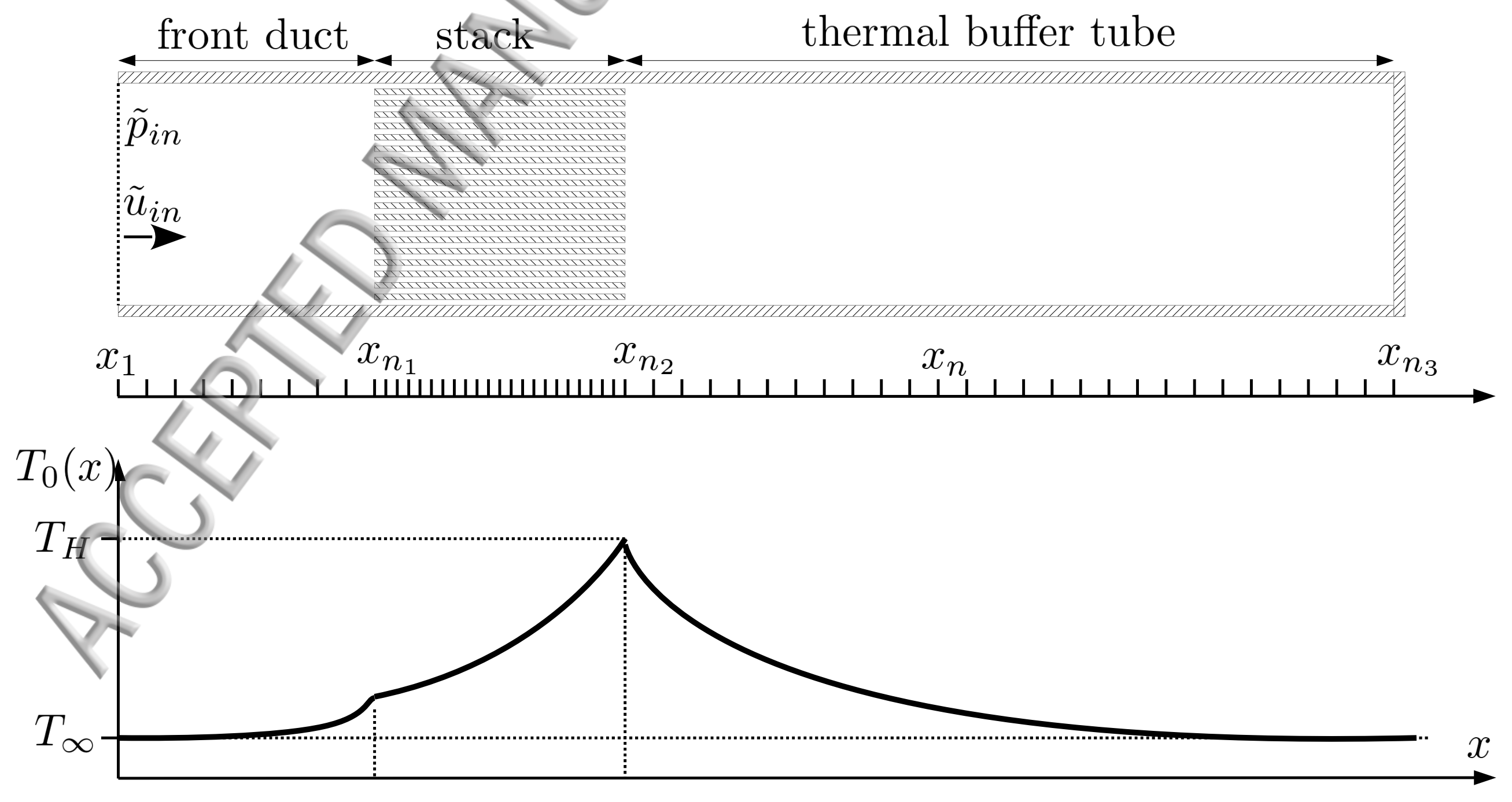




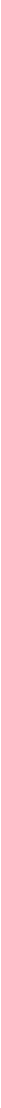




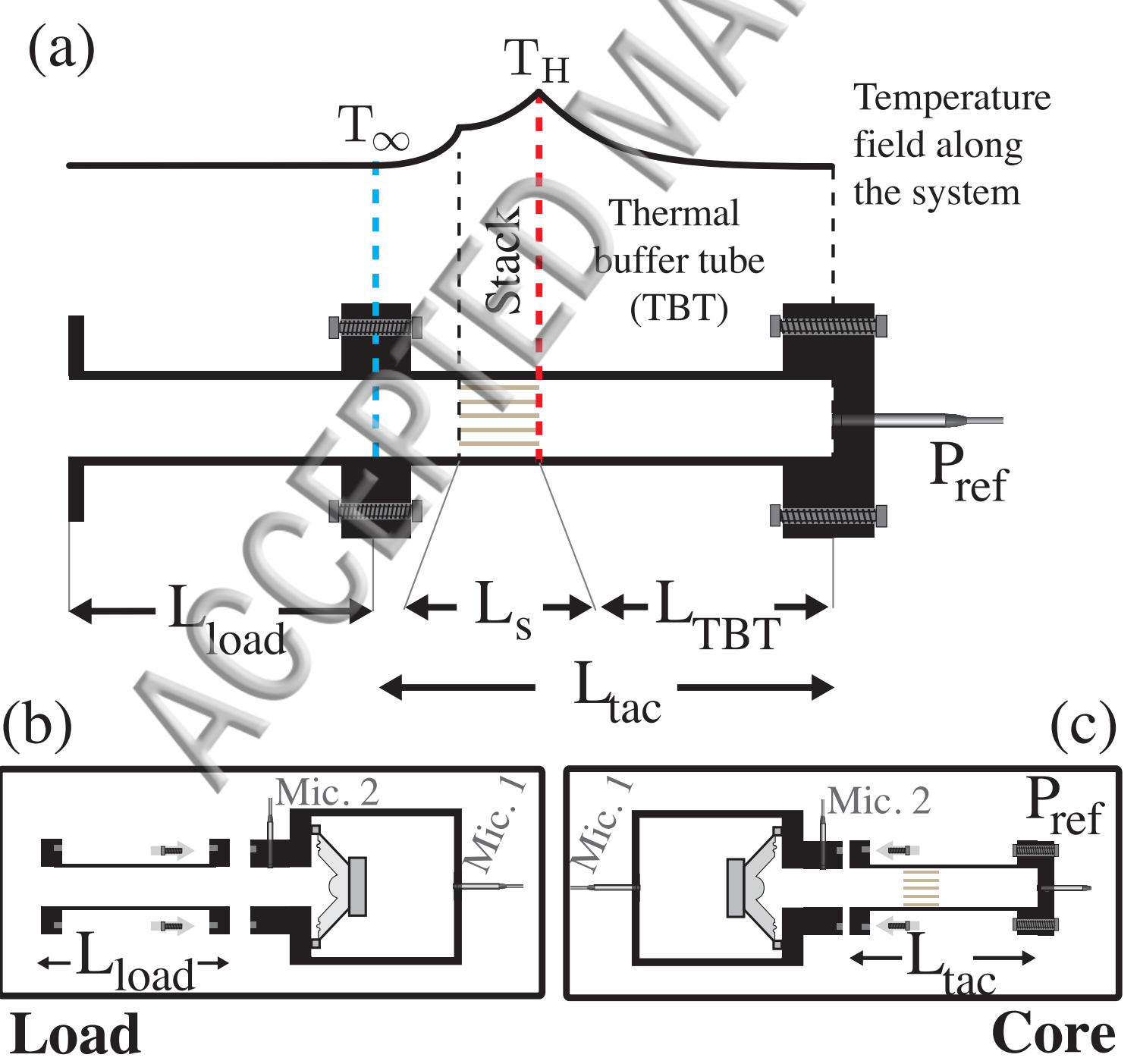




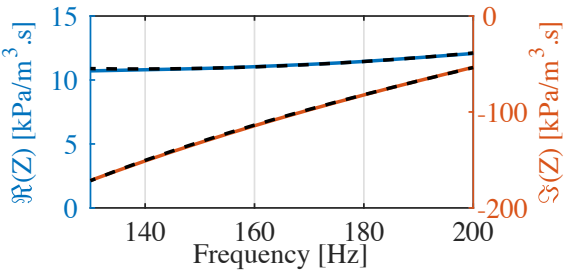




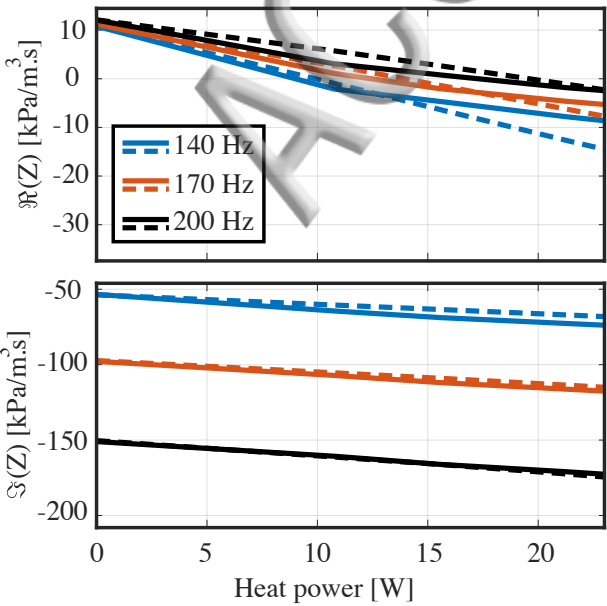




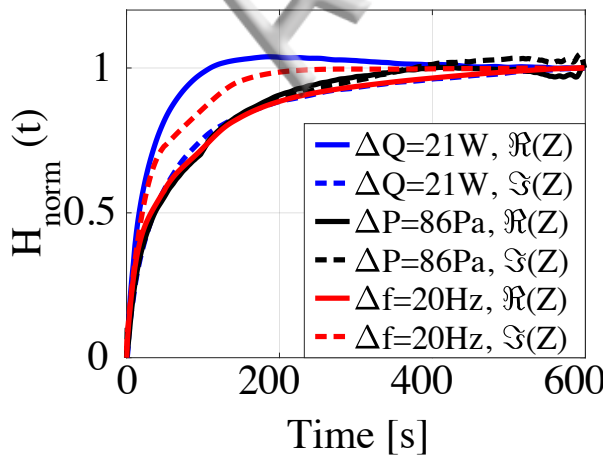




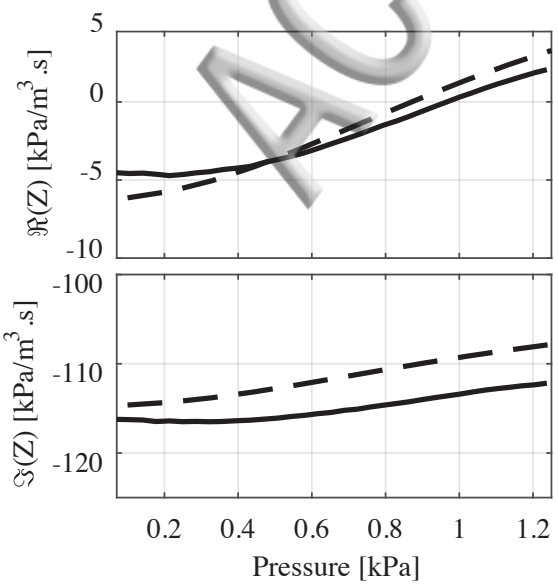




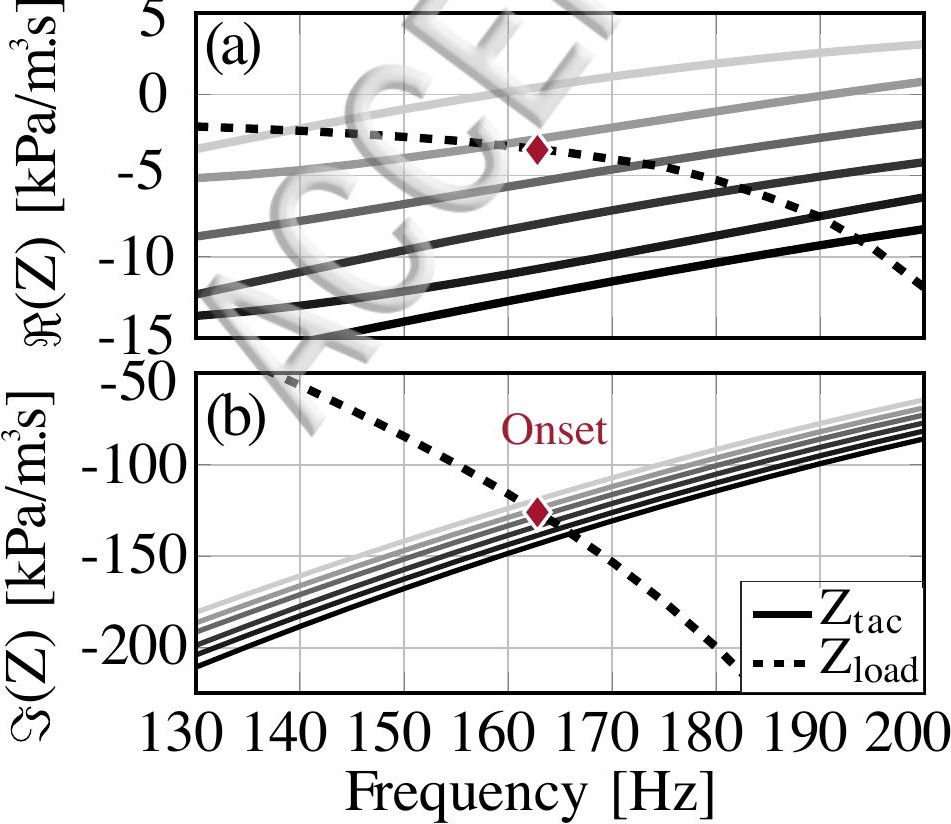


$\Re(\mathrm{Z})\left[\mathrm{kPa} / \mathrm{m}^{3} \mathrm{~s}\right]$

$\Im(\mathrm{Z})\left[\mathrm{kPa} / \mathrm{m}^{3} \mathrm{~s}\right]$

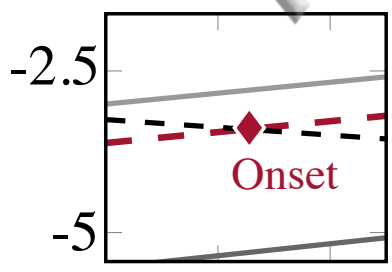

160162164

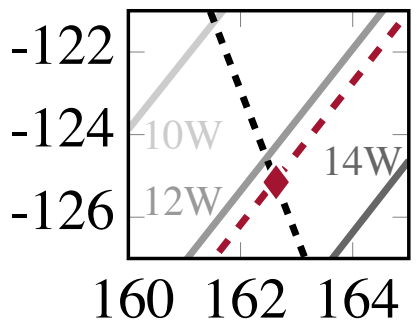

(d) Frequency $[\mathrm{Hz}]$ 


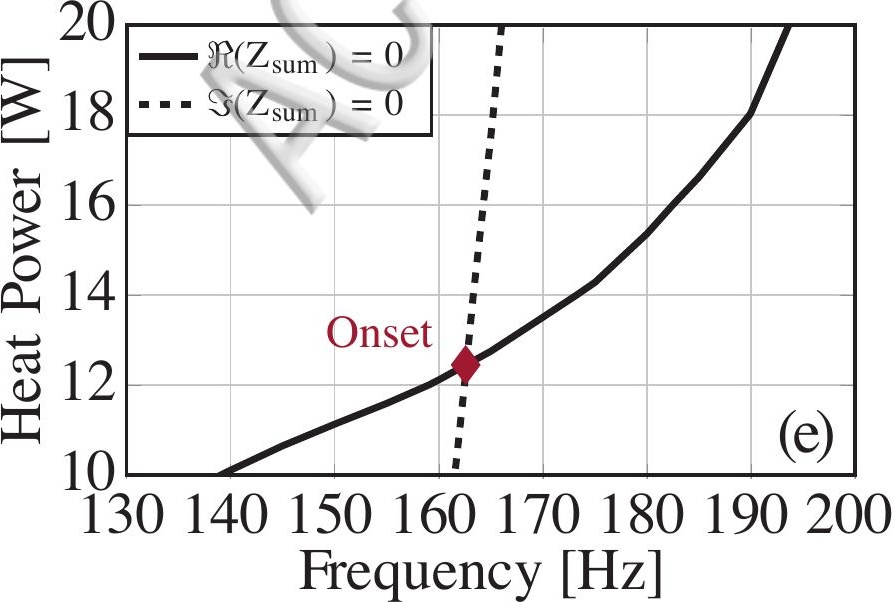




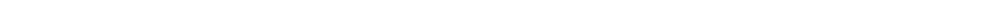


